TITLE:

\title{
Ab initio calculation of proton- coupled electron transfer rates using the external-potential representation: A ubiquinol complex in solution
}

\author{
$\operatorname{AUTHOR}(\mathrm{S}):$ \\ Yamamoto, T; Kato, S
}

\section{CITATION:}

Yamamoto, T ... [et al]. Ab initio calculation of proton-coupled electron transfer rates using the external-potential representation: A ubiquinol complex in solution. JOURNAL OF CHEMICAL PHYSICS 2007, 126(22): 224514.

ISSUE DATE:

2007-06-14

URL:

http://hdl.handle.net/2433/50585

\section{RIGHT:}

Copyright 2007 American Institute of Physics. This article may be downloaded for personal use only. Any other use requires prior permission of the author and the American Institute of Physics. 


\title{
$A b$ initio calculation of proton-coupled electron transfer rates using the external-potential representation: A ubiquinol complex in solution
}

\author{
Takeshi Yamamoto $^{\text {a) }}$ and Shigeki Kato ${ }^{\text {b) }}$ \\ Department of Chemistry, Graduate School of Science, Kyoto University, Kyoto 606-8502, Japan
}

(Received 9 February 2007; accepted 12 April 2007; published online 13 June 2007)

\begin{abstract}
In quantum-mechanical/molecular-mechanical $(\mathrm{QM} / \mathrm{MM})$ treatment of chemical reactions in condensed phases, one solves the electronic Schrödinger equation for the solute (or an active site) under the electrostatic field from the environment. This Schrödinger equation depends parametrically on the solute nuclear coordinates $R$ and the external electrostatic potential $V$. This fact suggests that one may use $R$ and $V$ as natural collective coordinates for describing the entire system, where $V$ plays the role of collective solvent variables. In this paper such an $(R, V)$ representation of the QM/MM canonical ensemble is described, with particular focus on how to treat charge transfer processes in this representation. As an example, the above method is applied to the proton-coupled electron transfer of a ubiquinol analog with phenoxyl radical in acetonitrile solvent. $A b$ initio free-energy surfaces are calculated as functions of $R$ and $V$ using the reference interaction site model self-consistent field method, the equilibrium points and the minimum free-energy crossing point are located in the $(R, V)$ space, and then the kinetic isotope effects (KIEs) are evaluated approximately. The results suggest that a stiffer proton potential at the transition state may be responsible for unusual KIEs observed experimentally for related systems. (C) 2007 American Institute of Physics. [DOI: 10.1063/1.2737048]
\end{abstract}

\section{INTRODUCTION}

Accurate $a b$ initio treatment of chemical reactions in solution and biological systems is a challenging task in theoretical chemistry. A very useful approach for this purpose are the so-called quantum-mechanical/molecular-mechanical (QM/MM) methods, where one treats the electronic structure of the solute (or an active site) with quantum-chemical methods and the rest of the system with empirical force fields. In the former calculation, one solves the following electronic Schrödinger equation for the solute:

$$
\left[\hat{H}_{0}+\hat{\mathbf{Q}} \cdot \mathbf{V}\right] \Psi=E \Psi,
$$

where $\mathbf{V}$ is the external electrostatic potential acting on solute atomic sites (here, we employ a site-site representation for solute-solvent electrostatic interaction; see Sec. II). The above Schrödinger equation depends parametrically on the solute nuclear coordinates (denoted as $\mathbf{R}_{u}$ ) and the external potential $\mathbf{V}$. This fact suggests that one may use $\mathbf{R}_{u}$ and $\mathbf{V}$ as natural collective coordinates for describing the entire system, where $\mathbf{V}$ plays the role of collective solvent coordinates. This type of idea has been utilized by several authors in the literature, ${ }^{1-8}$ including recent work by $\mathrm{Lu}$ and $\mathrm{Yang}^{6,7}$ for constructing a reaction-path potential for enzyme reactions, and also by Yamazaki and $\mathrm{Kato}^{8}$ for locating conical intersections of photochemical reactions in solution. In this paper we describe such an $\left(\mathbf{R}_{u}, \mathbf{V}\right)$ representation of the canonical ensemble based on the QM/MM framework, with particular emphasis on its kinetic aspects. The central quantity here is a

\footnotetext{
${ }^{a)}$ Electronic mail: yamamoto@kuchem.kyoto-u.ac.jp

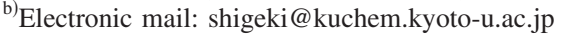

$V$-resolved free energy, $F\left(\mathbf{R}_{u}, \mathbf{V}\right)$, which relates to the probability density for observing a particular value of the solvent electrostatic potential $\mathbf{V}$. By construction, this $V$-resolved free energy can be separated neatly into the electronic energy of the quantum solute and the statistical self-energy (or freeenergy cost) of the classical solvent. Similar decomposition of the nonequilibrium solvation free energy is well-known for the quantum solute embedded in a dielectric continuum. $^{9-13}$ Our particular interest here is how to describe charge transfer reactions in this $\left(\mathbf{R}_{u}, \mathbf{V}\right)$ representation. To this end, we discuss in detail how one can transform the standard rate expressions for nonadiabatic electron transfer (including the mixed quantum/classical case) from Cartesian to the $\left(\mathbf{R}_{u}, \mathbf{V}\right)$ representation. Interestingly, the resulting rate expressions take a form very similar to those in Cartesian space, and also provide some rationale for quantizing a proton directly on a classical free-energy surface (Sec. II D 2).

Our second aim is to apply the above method to a proton-coupled electron transfer (PCET) reaction. PCET usually refers to a process where an active electron transfers between two different $\pi$ orbitals of electron donor-acceptor groups, while a proton transfers between proton donoracceptor sites due to the associated change in the diabatic potential energy (see Refs. 14 and 15 for recent reviews). Concerted PCET reactions can proceed more preferentially than single proton or single electron transfer due to a lower activation barrier of the former. ${ }^{14}$ This type of reaction is thought to play an important role in many biological processes, including photosynthesis, respiration, and DNA replication and repair. ${ }^{15}$ In order to avoid inherent complexity of biological processes, model synthetic systems have been investigated to obtain mechanistic insights into PCET 
reactions. ${ }^{15}$ Theoretical studies are still limited compared to the usual hydrogen transfer reactions, but representative work includes the quantum-chemical calculations by Mayer et $a{ }^{16}{ }^{16}$ and by Skone et al. ${ }^{17}$ that revealed an important difference in electronic structure between PCET and hydrogen atom transfer (HAT) pathways, and the reaction rate theory developed by Cukier, ${ }^{18-20}$ and Soudackov and Hammes-Schiffer ${ }^{21-23}$ based on a dielectric continuum model (see also a recent molecular-dynamics based approach in Ref. 24).

In this paper we study the PCET reaction of a ubiquinol analog with phenoxyl radical in acetonitrile using the $V$ representation mentioned above. Quinone and quinol are "shuttles" of electrons and protons over different components of cell membrane, and ubiquinol is a doubly reduced form of ubiquinone that can be found ubiquitously in mammalian cells. Our motivation for choosing this particular system is twofold: first, Mayer et al. ${ }^{16}$ predicted that the hydrogen exchange reaction between phenol and phenoxyl radical in the gas phase proceeds via PCET rather than HAT mechanism due to the lower activation barrier of the former. Second, a recent experiment by Cape et al. ${ }^{25}$ revealed that the apparent activation energy $\Delta G^{\ddagger}$ for the PCET reaction of ubiquinol with a poly-pyridyl ruthenium complex in acetonitrile exhibits an inverted relation for the proton $(H)$ and deuteron $(D)$ transfer, namely $\Delta G^{\ddagger}(H)>\Delta G^{\ddagger}(D)$. An analogous inverted kinetic isotope effect (KIE) was also observed by Nagaoka et l. $^{26}$ for the reaction of ubiquinol with a tocopherol derivative in ethanol (a model reaction of antioxidative radical trapping in biomembrane). The primary mechanism for these unusual KIE is still unknown, though relevant theoretical arguments have been made in the literature. ${ }^{27}$

The outline of this paper is as follows: In Sec. II we describe the $(R, V)$ representation of the QM/MM canonical ensemble and consider how to treat charge transfer processes in this representation. Approximate ways for calculating $V$-resolved free energy are discussed in Appendix A. In Sec. III we apply the above method to the PCET reaction of a ubiquinol complex in solution, where we utilize the reference interaction site model self-consistent field (RISM-SCF) (Refs. 28 and 29)/second-order Møller-Plesset perturbation (MP2) method. In Sec. IV we conclude by mentioning some technical issues to be addressed.

\section{CHARGE TRANSFER RATES IN THE EXTERNAL- POTENTIAL REPRESENTATION}

\section{A. $A b$ initio treatment of the solute in solution}

In this paper we assume a QM/MM model for the solution with a nonpolarizable MM force field for the solvent. Then, the potential energy of the total system may be written as

$$
\begin{aligned}
U[\mathbf{R} ; \Psi]= & \left\langle\Psi\left|\hat{H}_{0}\right| \Psi\right\rangle+\mathbf{Q}[\Psi] \cdot \mathbf{V}(\mathbf{R}) \\
& +U_{u v}^{\mathrm{LJ}}(\mathbf{R})+U_{v v}\left(\mathbf{R}_{v}\right),
\end{aligned}
$$

where we denote the Cartesian coordinates of the solute, solvent, and the total system as $\mathbf{R}_{u}, \mathbf{R}_{v}$, and $\mathbf{R}=\left(\mathbf{R}_{u}, \mathbf{R}_{v}\right)$, respectively. $U_{u v}^{\mathrm{LJ}}(\mathbf{R})$ is the short-range solute-solvent interac- tion (typically of the Lennard-Jones form) and $U_{v v}\left(\mathbf{R}_{v}\right)$ is the potential energy of the bulk solvent (including both shortand long-range interactions). $\hat{H}_{0}$ and $\Psi$ are the solute electronic Hamiltonian in the gas phase and the solute electronic wave function, respectively. (For notational simplicity, we will not denote the parametric dependence of $\hat{H}_{0}$ and $\Psi$ on $\mathbf{R}_{u}$ explicitly.) Note that $\Psi$ in Eq. (2) is subject to the electrostatic field produced by the solvent, which is denoted here as

$$
\mathbf{V}(\mathbf{R})=\left(V\left(\mathbf{R}_{u, 1} ; \mathbf{R}_{v}\right), \ldots, V\left(\mathbf{R}_{u, N} ; \mathbf{R}_{v}\right)\right),
$$

with

$$
V\left(\mathbf{x} ; \mathbf{R}_{v}\right)=\sum_{s}^{\text {solvent }} \frac{q_{s}}{\left|\mathbf{x}-\mathbf{R}_{v, s}\right|},
$$

where $\left\{\mathbf{R}_{u, k}\right\}$ and $\left\{\mathbf{R}_{v, s}\right\}$ are solute and solvent atomic sites, respectively, and $\left\{q_{s}\right\}$ is partial charges associated with the solvent sites. The external potential $\mathbf{V}(\mathbf{R})$ is coupled with partial charges of the solute, $\mathbf{Q}[\Psi]=\left(Q_{1}[\Psi], \ldots, Q_{N}[\Psi]\right)$, as in Eq. (2), where the partial charges are functionals of the solute wave function $\Psi$. In this paper we employ an electrostatic-potential-based definition of partial charges, which allows a compact operator notation as ${ }^{30-33}$

$$
\mathbf{Q}[\Psi]=\langle\Psi|\hat{\mathbf{Q}}| \Psi\rangle
$$

where $\hat{\mathbf{Q}}$ is a type of one-electron operator that generates partial charges on solute atomic sites. To proceed further, it is convenient to define a solvated electronic Schrödinger equation of the form

$$
\left[\hat{H}_{0}+\hat{\mathbf{Q}} \cdot \mathbf{V}^{\prime}\right] \Psi\left(\mathbf{R}_{u}, \mathbf{V}^{\prime}\right)=E\left(\mathbf{R}_{u}, \mathbf{V}^{\prime}\right) \Psi\left(\mathbf{R}_{u}, \mathbf{V}^{\prime}\right),
$$

where $\mathbf{V}^{\prime}$ is an arbitrary input parameter (in the same sense as the solute nuclear coordinates $\left.\mathbf{R}_{u}\right) . E\left(\mathbf{R}_{u}, \mathbf{V}^{\prime}\right)$ will be called the solvated electronic energy of the solute, and it represents the sum of the solute bare electronic energy and the solute-solvent electrostatic coupling, i.e.,

$$
E\left(\mathbf{R}_{u}, \mathbf{V}^{\prime}\right)=\left\langle\Psi\left(\mathbf{V}^{\prime}\right)\left|\hat{H}_{0}\right| \Psi\left(\mathbf{V}^{\prime}\right)\right\rangle+\mathbf{Q}\left[\Psi\left(\mathbf{V}^{\prime}\right)\right] \cdot \mathbf{V}^{\prime},
$$

where we abbreviate $\Psi\left(\mathbf{R}_{u}, \mathbf{V}^{\prime}\right)$ as $\Psi\left(\mathbf{V}^{\prime}\right)$. The solute wave function under the instantaneous electrostatic field from the solvent $\mathbf{V}(\mathbf{R})$ can then be written as $\Psi(\mathbf{V}(\mathbf{R}))$, and the QM/MM potential energy in Eq. (2) becomes

$$
U[\mathbf{R} ; \Psi(\mathbf{V}(\mathbf{R}))]=E\left(\mathbf{R}_{u}, \mathbf{V}(\mathbf{R})\right)+U_{u v}^{\mathrm{LJ}}(\mathbf{R})+U_{v v}\left(\mathbf{R}_{v}\right) .
$$

Note that $U_{u v}^{\mathrm{LJ}}(\mathbf{R})$ and $U_{v v}\left(\mathbf{R}_{v}\right)$ do not depend on the solute wave function in the present model.

\section{B. $V$-resolved free energy}

The central quantity in the following discussions is the $V$-resolved free energy, $F\left(\mathbf{R}_{u}, \mathbf{V}^{\prime}\right)$, which is defined by inserting the resolution of unity $1=\int d \mathbf{V}^{\prime} \delta\left(\mathbf{V}^{\prime}-\mathbf{V}(\mathbf{R})\right)$ into the (configurational) partition function as ${ }^{34}$ 


$$
\begin{aligned}
Z & =\int d \mathbf{R}_{u} \int d \mathbf{R}_{v} e^{-\beta U[\mathbf{R} ; \Psi(\mathbf{V}(\mathbf{R}))]} \\
& =Z_{v} \int d \mathbf{R}_{u} \int d \mathbf{V}^{\prime} e^{-\beta F\left(\mathbf{R}_{u}, \mathbf{V}^{\prime}\right)},
\end{aligned}
$$

with

$$
e^{-\beta F\left(\mathbf{R}_{u}, \mathbf{V}^{\prime}\right)}=\frac{1}{Z_{v}} \int d \mathbf{R}_{v} e^{-\beta U[\mathbf{R} ; \Psi(\mathbf{V}(\mathbf{R}))]} \delta\left(\mathbf{V}^{\prime}-\mathbf{V}(\mathbf{R})\right),
$$

where $Z_{v}$ is the partition function of the bulk solvent, ${ }^{35} Z_{v}$ $=\int d \mathbf{R}_{v} \exp \left[-\beta U_{v v}\left(\mathbf{R}_{v}\right)\right]$. The above $F\left(\mathbf{R}_{u}, \mathbf{V}^{\prime}\right)$ may be viewed as the solvation free energy of the quantum solute with a fixed geometry $\mathbf{R}_{u}$ under the additional constraint that $\mathbf{V}(\mathbf{R})=\mathbf{V}^{\prime}$. Due to the presence of $\delta\left(\mathbf{V}^{\prime}-\mathbf{V}(\mathbf{R})\right)$, one can replace $\mathbf{V}(\mathbf{R})$ in Eq. (10) with $\mathbf{V}^{\prime}$ and extract the solvated electronic energy $E\left(\mathbf{R}_{u}, \mathbf{V}^{\prime}\right)$ as

$e^{-\beta F\left(\mathbf{R}_{u}, \mathbf{V}^{\prime}\right)}=e^{-\beta E\left(\mathbf{R}_{u}, \mathbf{V}^{\prime}\right)} \frac{1}{Z_{v}} \int d \mathbf{R}_{v} e^{-\beta U_{0}(\mathbf{R})} \delta\left(\mathbf{V}^{\prime}-\mathbf{V}(\mathbf{R})\right)$,

where $U_{0}(\mathbf{R})$ is the short-range solute-solvent interaction plus the bulk solvent potential,

$$
U_{0}(\mathbf{R})=U_{u v}^{\mathrm{LJ}}(\mathbf{R})+U_{v v}\left(\mathbf{R}_{v}\right) .
$$

By introducing the solvent self-energy $S_{0}\left(\mathbf{R}_{u}, \mathbf{V}^{\prime}\right)$ defined by

$$
e^{-\beta S_{0}\left(\mathbf{R}_{u}, \mathbf{V}^{\prime}\right)}=\frac{1}{Z_{v}} \int d \mathbf{R}_{v} e^{-\beta U_{0}(\mathbf{R})} \delta\left(\mathbf{V}^{\prime}-\mathbf{V}(\mathbf{R})\right),
$$

one can rewrite Eq. (11) compactly as

$$
F\left(\mathbf{R}_{u}, \mathbf{V}^{\prime}\right)=E\left(\mathbf{R}_{u}, \mathbf{V}^{\prime}\right)+S_{0}\left(\mathbf{R}_{u}, \mathbf{V}^{\prime}\right) .
$$

That is, $F\left(\mathbf{R}_{u}, \mathbf{V}^{\prime}\right)$ is determined by the balance of two terms, namely (i) the electronic energy $E\left(\mathbf{R}_{u}, \mathbf{V}^{\prime}\right)$ of the quantum solute that includes all the solute-solvent electrostatic interactions, and (ii) the self-energy $S_{0}\left(\mathbf{R}_{u}, \mathbf{V}^{\prime}\right)$ that describes the free-energy cost for the classical solvent to produce $\mathbf{V}^{\prime}$ around an uncharged solute. In Appendix A we describe approximate ways for calculating $F\left(\mathbf{R}_{u}, \mathbf{V}\right)$ based on the Gaussian fluctuation model for the solvent. ${ }^{1,2}$

\section{Electron transfer rate in the classical nuclear limit}

We now transform the standard rate expression for nonadiabatic electron transfer (ET) in the classical nuclear limit from Cartesian to the $\left(\mathbf{R}_{u}, \mathbf{V}^{\prime}\right)$ representation (see Sec. II D for the quantization of light atoms). The diabatic wave functions involved in the ET reaction are denoted as $\Psi_{\mathrm{I}}$ and $\Psi_{\mathrm{II}}$, with their electronic coupling given by $V_{\mathrm{el}}$. We calculate the transition rate from $\Psi_{\mathrm{I}}$ to $\Psi_{\mathrm{II}}$ with the following golden rule expression: ${ }^{36}$

$$
k_{\mathrm{ET}}=\kappa \frac{1}{Z_{\mathrm{I}}} \int d \mathbf{R} e^{-\beta U_{\mathrm{I}}(\mathbf{R})} \delta\left[U_{\mathrm{I}}(\mathbf{R})-U_{\mathrm{II}}(\mathbf{R})\right],
$$

where $U_{K}(\mathbf{R})=U\left[\mathbf{R} ; \Psi_{K}(\mathbf{V}(\mathbf{R}))\right](K=\mathrm{I}, \mathrm{II})$ is the potential energy corresponding to each diabatic state, $Z_{I}$ is the reactant partition function $Z_{I}=\int d \mathbf{R} \exp \left[-\beta U_{\mathrm{I}}(\mathbf{R})\right]$, and $\kappa$ is the prefactor given by $(2 \pi / \hbar) V_{\mathrm{el}}^{2}$. Here, the nuclear dependence of $V_{\text {el }}$ has been neglected (the Condon approximation). In the above golden rule approximation, electronic transitions occur only on the crossing seam between diabatic potential-energy surfaces, namely $U_{\mathrm{I}}(\mathbf{R})=U_{\mathrm{II}}(\mathbf{R})$. The usual method for evaluating Eq. (15) is to insert the resolution of unity 1 $=\int d \Delta U^{\prime} \delta\left[U_{\mathrm{I}}(\mathbf{R})-U_{\mathrm{II}}(\mathbf{R})-\Delta U^{\prime}\right]$ into the integrand of Eq. (15) to obtain ${ }^{37-44}$

$$
k_{\mathrm{ET}}=\kappa \frac{1}{Z_{\mathrm{I}} / Z_{v}} \int d \Delta U^{\prime} \delta\left(\Delta U^{\prime}\right) e^{-\beta G_{\mathrm{I}}\left(\Delta U^{\prime}\right)},
$$

where the energy-gap free-energy $G_{K}\left(\Delta U^{\prime}\right)$ for each diabatic state $(K=\mathrm{I}, \mathrm{II})$ is defined by

$e^{-\beta G_{K}\left(\Delta U^{\prime}\right)}=\frac{1}{Z_{v}} \int d \mathbf{R} e^{-\beta U_{K}(\mathbf{R})} \delta\left[U_{\mathrm{I}}(\mathbf{R})-U_{\mathrm{II}}(\mathbf{R})-\Delta U^{\prime}\right]$.

The above free energy can be evaluated using the standard histogram method, and in many cases $G_{K}\left(\Delta U^{\prime}\right)$ becomes a quadratic function of $\Delta U^{\prime}$ to a very good approximation. Using the relation $G_{\mathrm{I}}\left(\Delta U^{\prime}\right)=G_{\mathrm{II}}\left(\Delta U^{\prime}\right)+\Delta U^{\prime}$ that holds rigorously by definition, one can rewrite Eq. (16) as

$$
\begin{aligned}
k_{\mathrm{ET}}= & \kappa \frac{1}{Z_{\mathrm{I}} / Z_{v}} \int d \Delta U^{\prime} e^{-\beta G_{\mathrm{I}}\left(\Delta U^{\prime}\right)} \\
& \times \delta\left[G_{\mathrm{I}}\left(\Delta U^{\prime}\right)-G_{\mathrm{II}}\left(\Delta U^{\prime}\right)\right],
\end{aligned}
$$

which allows an interpretation that electron transfer occurs at the crossing point of two free-energy curves, $G_{\mathrm{I}}\left(\Delta U^{\prime}\right)$ and $G_{\mathrm{II}}\left(\Delta U^{\prime}\right)$.

One can proceed in a similar manner when one chooses the solvent electrostatic potential $\mathbf{V}(\mathbf{R})$ as collective solvent coordinates. That is, one inserts the resolution of unity as

$$
\begin{aligned}
k_{\mathrm{ET}}= & \kappa \frac{1}{Z_{\mathrm{I}}} \int d \mathbf{R} \int d \mathbf{V}^{\prime} e^{-\beta U_{\mathrm{I}}(\mathbf{R})} \\
& \times \delta\left[U_{\mathrm{I}}(\mathbf{R})-U_{\mathrm{II}}(\mathbf{R})\right] \delta\left(\mathbf{V}^{\prime}-\mathbf{V}(\mathbf{R})\right),
\end{aligned}
$$

and, using the following relation [cf. Eq. (8)]:

$$
U_{\mathrm{I}}(\mathbf{R})-\left.U_{\mathrm{II}}(\mathbf{R})\right|_{\mathbf{V}(\mathbf{R})=\mathbf{V}^{\prime}}=E_{\mathrm{I}}\left(\mathbf{R}_{u}, \mathbf{V}^{\prime}\right)-E_{\mathrm{II}}\left(\mathbf{R}_{u}, \mathbf{V}^{\prime}\right),
$$

one rewrites Eq. (19) as

$$
\begin{aligned}
k_{\mathrm{ET}}= & \kappa \frac{1}{Z_{\mathrm{I}} / Z_{v}} \int d \mathbf{R}_{u} \int d \mathbf{V}^{\prime} e^{-\beta F_{\mathrm{I}}\left(\mathbf{R}_{u}, \mathbf{V}^{\prime}\right)} \\
& \times \delta\left[E_{\mathrm{I}}\left(\mathbf{R}_{u}, \mathbf{V}^{\prime}\right)-E_{\mathrm{II}}\left(\mathbf{R}_{u}, \mathbf{V}^{\prime}\right)\right],
\end{aligned}
$$

where $E_{K}\left(\mathbf{R}_{u}, \mathbf{V}^{\prime}\right)$ and $F_{K}\left(\mathbf{R}_{u}, \mathbf{V}^{\prime}\right)$ are diabatic counterparts of $E\left(\mathbf{R}_{u}, \mathbf{V}^{\prime}\right)$ and $F\left(\mathbf{R}_{u}, \mathbf{V}^{\prime}\right)$ defined in the previous section. The matching condition of the total potential energies in Eq. (15) is thus recast into that of the solvated electronic energies, $E_{\mathrm{I}}=E_{\mathrm{II}}$. One can further rewrite the above equation as 


$$
\begin{aligned}
k_{\mathrm{ET}}= & \kappa \frac{1}{Z_{\mathrm{I}} / Z_{v}} \int d \mathbf{R}_{u} \int d \mathbf{V}^{\prime} e^{-\beta F_{\mathrm{I}}\left(\mathbf{R}_{u}, \mathbf{V}^{\prime}\right)} \\
& \times \delta\left[F_{\mathrm{I}}\left(\mathbf{R}_{u}, \mathbf{V}^{\prime}\right)-F_{\mathrm{II}}\left(\mathbf{R}_{u}, \mathbf{V}^{\prime}\right)\right],
\end{aligned}
$$

by adding the self-energy term $S_{0}\left(\mathbf{R}_{u}, \mathbf{V}^{\prime}\right)$ to both $E_{\mathrm{I}}$ and $E_{\mathrm{II}}$ in the delta function (note that the self-energy does not depend on $\Psi_{K}$ ). The last equation is interesting in that the electronic transition could be viewed as occurring between two free-energy surfaces, $F_{\mathrm{I}}$ and $F_{\mathrm{II}}$, in the reduced $\left(\mathbf{R}_{u}, \mathbf{V}^{\prime}\right)$ space. Free-energy-based expressions such as Eq. (22) may be useful when one wishes to avoid explicit reference to the underlying potential energy (e.g., when one invokes an implicit solvent model). Of course, this does not mean that one can unconditionally interpret a free energy as an effective potential energy. For example, it is a highly nontrivial matter whether or under what condition one may quantize a proton directly on a classical free-energy surface (see Sec. II D 2).

\section{Proton-coupled electron transfer rate}

When light atoms play important roles in a reaction, e.g., in the case of proton-coupled electron transfer (PCET), it is desirable to take appropriate account of nuclear quantum effects. A useful starting point to that end is the following mixed quantum-classical rate expression (again within the golden rule approximation): ${ }^{36}$

$$
\begin{aligned}
k_{\mathrm{ET}}= & \kappa \frac{1}{Z_{\mathrm{I}}} \sum_{m} \sum_{n} \int d \tilde{\mathbf{R}} e^{-\beta U_{\mathrm{II}}(\tilde{\mathbf{R}})}\left|T_{m n}(\tilde{\mathbf{R}})\right|^{2} \\
& \times \delta\left[U_{\mathrm{Im}}(\tilde{\mathbf{R}})-U_{\mathrm{II} n}(\tilde{\mathbf{R}})\right],
\end{aligned}
$$

where $\tilde{\mathbf{R}}$ is the classical (Cartesian) coordinates of the entire system except for the proton, and $U_{K l}(\tilde{\mathbf{R}})(K=\mathrm{I}, \mathrm{II})$ is the eigenenergy of the following nuclear Schrödinger equation for the proton:

$$
\left[\hat{T}_{p}+U_{K}(\mathbf{R})\right] \varphi_{K l}\left(\mathbf{r}_{p} ; \tilde{\mathbf{R}}\right)=U_{K l}(\tilde{\mathbf{R}})_{\varphi_{K l}}\left(\mathbf{r}_{p} ; \tilde{\mathbf{R}}\right) .
$$

Here, $U_{K}(\mathbf{R})$ is the classical diabatic potential energy, $\mathbf{r}_{p}$ is the (three-dimensional) proton coordinates, and $\hat{T}_{p}$ is the kinetic energy operator for $\mathbf{r}_{p} . T_{m n}(\tilde{\mathbf{R}})$ is the Franck-Condon factor given by $T_{m n}(\widetilde{\mathbf{R}})=\left\langle\varphi_{\mathrm{I} m}(\widetilde{\mathbf{R}}) \mid \varphi_{\mathrm{II} n}(\widetilde{\mathbf{R}})\right\rangle$. The rate expression in Eq. (23) describes the sum of transitions from the $m$ th reactant vibronic state to the $n$th product vibronic state. Our goal in this section is to transform this rate expression from Cartesian to the $V$ representation, as carried out in Sec. II C. In what follows, we first discuss a rigorous (but somewhat cumbersome) transformation and then proceed to a more approximate (thus much simpler) method for practical purposes.

\section{Rigorous transformation using the discrete variable representation}

In order to transform Eq. (23) rigorously to the $V$-representation, it is convenient to go back to the original golden rule expression that underlies Eq. (23) (i.e., that before taking the classical limit of $\tilde{\mathbf{R}}$ ),

$$
k_{\mathrm{ET}}=\left.\kappa \frac{1}{Q_{\mathrm{I}}} \sum_{a} \sum_{b} e^{-\beta W_{\mathrm{I} a} \mid}\left\langle\chi_{\mathrm{I} a} \mid \chi_{\mathrm{II} b}\right\rangle\right|^{2} \delta\left(W_{\mathrm{I} a}-W_{\mathrm{II} b}\right),
$$

where $\chi_{K l}$ and $W_{K l}$ are the eigenpair of the nuclear Schrödinger equation in the entire $\mathbf{R}$ space, i.e., $\hat{H}_{K \chi K l}$ $=W_{K l_{\chi K l}}$ with $\hat{H}_{K}=\hat{T}_{R}+U_{K}(\mathbf{R}) \cdot Q_{\mathrm{I}}$ is the reactant partition function, $Q_{\mathrm{I}}=\Sigma_{a} e^{-\beta W_{\mathrm{I} a}}$. It is easy to rewrite the above equation to a time-dependent form,

$$
k_{\mathrm{ET}}=\kappa \frac{1}{Q_{\mathrm{I}}} \int \frac{d t}{2 \pi \hbar} \operatorname{tr}_{R}\left[e^{-\beta \hat{H}_{\mathrm{I}}} e^{i \hat{H}_{\mathrm{II}} t / \hbar} e^{-i \hat{H}_{\mathrm{I}} t / \hbar}\right] .
$$

We then take the classical limit of $\tilde{\mathbf{R}}$ via the following replacement: $:^{43,45,46}$

$$
\begin{aligned}
& \operatorname{tr}_{R} \rightarrow \operatorname{tr}_{p} \iint \frac{d \tilde{\mathbf{P}} d \tilde{\mathbf{R}}}{(2 \pi \hbar)^{L}}, \\
& \hat{H}_{K} \rightarrow \hat{T}_{p}+T_{R}+U_{K}(\mathbf{R}),
\end{aligned}
$$

where $\operatorname{tr}_{p}$ is the quantum trace over the proton degrees of freedom, $\widetilde{\mathbf{P}}$ is the conjugate momenta of $\widetilde{\mathbf{R}}$, and $L=\operatorname{dim} \widetilde{\mathbf{R}}$. To proceed further, we make a very minor approximation that the dependence of $U_{0}(\mathbf{R})$ in Eq. (12) on $\mathbf{r}_{p}$ can be neglected due to the small van der Waals radius of the proton. The above replacement in Eqs. (27a) and (27b) then gives

$$
\begin{aligned}
k_{\mathrm{ET}}= & \kappa \frac{1}{Z_{\mathrm{I}}} \int \frac{d t}{2 \pi \hbar} \int d \tilde{\mathbf{R}} e^{-\beta U_{0}(\tilde{\mathbf{R}})} \\
& \times \operatorname{tr}_{p}\left[e^{-\beta \hat{h}_{\mathrm{I}}(\mathbf{V}(\mathbf{R}))} e^{i \hat{h}_{\mathrm{II}}(\mathbf{V}(\mathbf{R})) t / \hbar} e^{-i \hat{h}_{\mathrm{I}}(\mathbf{V}(\mathbf{R})) t / \hbar}\right],
\end{aligned}
$$

where $\hat{h}_{K}(\mathbf{V}(\mathbf{R}))$ is the solvated protonic Hamiltonian defined by

$$
\hat{h}_{K}\left(\mathbf{V}^{\prime}\right)=\hat{T}_{p}+E_{K}\left(\mathbf{R}_{u}, \mathbf{V}^{\prime}\right)
$$

at $\mathbf{V}^{\prime}=\mathbf{V}(\mathbf{R})$, and $E_{K}\left(\mathbf{R}_{u}, \mathbf{V}^{\prime}\right)$ is the solute electronic energy in Eq. (7). Expanding the protonic Hamiltonian $\hat{h}_{K}(\mathbf{V}(\mathbf{R}))$ in terms of its eigenstates gives the mixed quantum-classical rate expression in Eq. (23), which is not convenient to transform to the $V$ representation. Inserting the resolution of unity $1=\int d \mathbf{V}^{\prime} \delta\left(\mathbf{V}^{\prime}-\mathbf{V}(\mathbf{R})\right)$ into Eq. (28) is not very helpful either because $\mathbf{r}_{p}$ is a quantum operator and the replacement of $\mathbf{V}(\mathbf{R})$ in $\hat{h}_{K}(\mathbf{V}(\mathbf{R}))$ by $\mathbf{V}^{\prime}$ is not allowed. This problem can be avoided, however, by expressing $\hat{h}_{K}(\mathbf{V}(\mathbf{R}))$ in the discrete variable representation (DVR) as follows:

$$
\begin{aligned}
\hat{h}_{K}(\mathbf{V}(\mathbf{R})) \rightarrow & \hat{h}_{K}^{\mathrm{DVR}}\left(\mathbf{V}_{Q}(\tilde{\mathbf{R}})\right) \\
\equiv & \sum_{i} \sum_{j}\left|\mathbf{r}_{p}^{(i)}\right\rangle\left[T_{i j}+\left.\delta_{i j} E_{K}\left(\mathbf{R}_{u}, \mathbf{V}(\mathbf{R})\right)\right|_{\mathbf{r}_{p}=\mathbf{r}_{p}^{(i)}}\right] \\
& \times\left\langle\mathbf{r}_{p}^{(j)}\right|,
\end{aligned}
$$

where $\left\{\mathbf{r}_{p}^{(i)}\right\}$ are DVR points expressed in terms of solute backbone coordinates $\tilde{\mathbf{R}}_{u}$ [defined via $\tilde{\mathbf{R}}=\left(\tilde{\mathbf{R}}_{u}, \mathbf{R}_{v}\right)$ ] and $\left\{T_{i j}\right\}$ is an appropriate kinetic energy matrix in the DVR. $\mathbf{V}_{Q}(\widetilde{\mathbf{R}})$ in Eq. (30) is a quantum version of $\mathbf{V}(\mathbf{R})$, which includes the external potential on all the DVR points of the proton, 
$V_{p}^{(i)}(\tilde{\mathbf{R}}) \equiv V\left(\mathbf{r}_{p}^{(i)} ; \mathbf{R}_{v}\right)(i=1, \ldots, M)$ in addition to those on the remaining solute sites $\tilde{\mathbf{V}}(\tilde{\mathbf{R}})$, i.e.,

$$
\mathbf{V}_{Q}(\tilde{\mathbf{R}})=\left(V_{p}^{(1)}, \ldots, V_{p}^{(M)}, \tilde{\mathbf{V}}\right)
$$

It is now straightforward to reduce the solvent Cartesian coordinates $\mathbf{R}_{v}$ to the external potential $\mathbf{V}_{Q}(\tilde{\mathbf{R}})$ by inserting the resolution of unity $1=\int d \mathbf{V}_{Q}^{\prime} \delta\left(\mathbf{V}_{Q}^{\prime}-\mathbf{V}_{Q}(\tilde{\mathbf{R}})\right)$ into Eq. (28) (after expressing $\hat{h}_{K}$ in the DVR), which gives

$$
\begin{aligned}
& k_{\mathrm{ET}}=\kappa \frac{1}{Z_{\mathrm{I}} / Z_{v}} \int \frac{d t}{2 \pi \hbar} \int d \tilde{\mathbf{R}}_{u} \int d \mathbf{V}_{Q}^{\prime} e^{-\beta S_{Q}\left(\tilde{\mathbf{R}}_{u}, \mathbf{V}_{Q}^{\prime}\right)} \\
& \times \operatorname{tr}_{p}\left[e^{-\beta \hat{h}_{\mathrm{I}}^{\mathrm{DVR}}\left(\mathbf{V}_{Q}^{\prime}\right)} e^{i \hat{h}_{\mathrm{II}}^{\mathrm{DVR}}\left(\mathbf{V}_{Q}^{\prime}\right) t / \hbar} e^{-i \hat{h}_{\mathrm{I}}^{\mathrm{DVR}}\left(\mathbf{V}_{Q}^{\prime}\right) t / \hbar}\right]
\end{aligned}
$$

where $S_{Q}\left(\tilde{\mathbf{R}}_{u}, \mathbf{V}_{Q}^{\prime}\right)$ is an analog of the self-energy defined by

$$
e^{-\beta S_{Q}\left(\tilde{\mathbf{R}}_{u}, \mathbf{V}_{Q}^{\prime}\right)}=\frac{1}{Z_{v}} \int d \mathbf{R}_{v} e^{-\beta U_{0}(\tilde{\mathbf{R}})} \delta\left(\mathbf{V}_{Q}^{\prime}-\mathbf{V}_{Q}(\tilde{\mathbf{R}})\right)
$$

Performing an eigenstate expansion of $\hat{h}_{K}^{\mathrm{DVR}}\left(\mathbf{V}_{Q}^{\prime}\right)$ via

$$
\hat{h}_{K}^{\mathrm{DVR}}\left(\mathbf{V}_{Q}^{\prime}\right) \varphi_{K l}\left(\mathbf{r}_{p} ; \tilde{\mathbf{R}}_{u}, \mathbf{V}_{Q}^{\prime}\right)=\epsilon_{K l}\left(\tilde{\mathbf{R}}_{u}, \mathbf{V}_{Q}^{\prime}\right) \varphi_{K l}\left(\mathbf{r}_{p} ; \tilde{\mathbf{R}}_{u}, \mathbf{V}_{Q}^{\prime}\right),
$$

we obtain a desired rate expression in the $V$ representation,

$$
\begin{aligned}
k_{\mathrm{ET}}= & \kappa \frac{1}{Z_{\mathrm{I}} / Z_{v}} \sum_{m} \sum_{n} \int d \tilde{\mathbf{R}}_{u} \\
& \times \int d \mathbf{V}_{Q}^{\prime} e^{-\beta\left[\epsilon_{\mathrm{I} m}\left(\tilde{\mathbf{R}}_{u}, \mathbf{V}_{Q}^{\prime}\right)+S_{Q}\left(\tilde{\mathbf{R}}_{u}, \mathbf{V}_{Q}^{\prime}\right)\right]} \\
& \times\left|T_{m n}\left(\tilde{\mathbf{R}}_{u}, \mathbf{V}_{Q}^{\prime}\right)\right|^{2} \delta\left[\epsilon_{\mathrm{I} m}\left(\tilde{\mathbf{R}}_{u}, \mathbf{V}_{Q}^{\prime}\right)-\epsilon_{\mathrm{II} n}\left(\tilde{\mathbf{R}}_{u}, \mathbf{V}_{Q}^{\prime}\right)\right],
\end{aligned}
$$

where $T_{m n}\left(\tilde{\mathbf{R}}_{u}, \mathbf{V}_{Q}^{\prime}\right)$ is the overlap between $\varphi_{\mathrm{I} m}$ and $\varphi_{\mathrm{II} n}$ at $\left(\tilde{\mathbf{R}}_{u}, \mathbf{V}_{Q}^{\prime}\right)$.

\section{Probe-point approximation}

Although the above DVR approach is rather rigorous, one can obtain a much simpler rate expression by approximating the external potential acting on the proton as

$$
V\left(\mathbf{r}_{p} ; \mathbf{R}_{v}\right) \simeq V\left(\mathbf{r}_{p}^{\#}\left(\tilde{\mathbf{R}}_{u}\right) ; \mathbf{R}_{v}\right),
$$

where $\mathbf{r}_{p}^{\#}\left(\tilde{\mathbf{R}}_{u}\right)$ is a "probe" point that evaluates the external field instead of the instantaneous position of the proton. In the simplest case, $\mathbf{r}_{p}^{\#}$ could be chosen roughly as the midpoint between the donor and acceptor atoms. The above approximation is based on the expectation that $V\left(\mathbf{r}_{p} ; \mathbf{R}_{v}\right)$ will not vary too rapidly as a function of $\mathbf{r}_{p}$ within a proton donor-acceptor interface (see Appendix B for an improved treatment). Once the probe-point approximation has been made, one can readily obtain a mixed quantum-classical rate expression in the $V$ representation by defining an approximate $V$-vector as

$$
\mathbf{V}(\mathbf{R}) \simeq \mathbf{V}^{\#}(\tilde{\mathbf{R}}) \equiv\left(V\left(\mathbf{r}_{p}^{\#} ; \mathbf{R}_{v}\right), \tilde{\mathbf{V}}(\tilde{\mathbf{R}})\right)
$$

replacing $\hat{h}_{K}(\mathbf{V}(\mathbf{R}))$ by $\hat{h}_{K}\left(\mathbf{V}^{\#}(\tilde{\mathbf{R}})\right)$ in Eq. (28), and then inserting the resolution of unity $1=\int d \mathbf{V}^{\prime} \delta\left(\mathbf{V}^{\prime}-\mathbf{V}^{\#}(\tilde{\mathbf{R}})\right)$ into Eq. (28) so that $\mathbf{V}^{\#}(\tilde{\mathbf{R}})$ in $\hat{h}_{K}\left(\mathbf{V}^{\#}(\tilde{\mathbf{R}})\right)$ may be replaced by $\mathbf{V}^{\prime}$ [note that $\mathbf{V}^{\#}(\tilde{\mathbf{R}})$ is no longer a quantum operator and thus the above replacement is valid]. The result is

$$
\begin{aligned}
& k_{\mathrm{ET}}=\kappa \frac{1}{Z_{\mathrm{I}} / Z_{v}} \sum_{m} \sum_{n} \int d \tilde{\mathbf{R}}_{u} \int d \mathbf{V}^{\prime} e^{-\beta\left[\epsilon_{\mathrm{I} m}\left(\tilde{\mathbf{R}}_{u}, \mathbf{V}^{\prime}\right)+S_{0}^{\#}\left(\tilde{\mathbf{R}}_{u}, \mathbf{V}^{\prime}\right)\right]} \\
& \times\left|T_{m n}\left(\tilde{\mathbf{R}}_{u}, \mathbf{V}^{\prime}\right)\right|^{2} \delta\left[\epsilon_{\mathrm{I} m}\left(\tilde{\mathbf{R}}_{u}, \mathbf{V}^{\prime}\right)-\epsilon_{\mathrm{II} n}\left(\tilde{\mathbf{R}}_{u}, \mathbf{V}^{\prime}\right)\right],
\end{aligned}
$$

where $S_{0}^{\#}\left(\tilde{\mathbf{R}}_{u}, \mathbf{V}^{\prime}\right)$ is a variant of the self-energy defined by

$$
e^{-\beta S_{0}^{\#}\left(\tilde{\mathbf{R}}_{u}, \mathbf{V}^{\prime}\right)}=\frac{1}{Z_{v}} \int d \mathbf{R}_{v} e^{-\beta U_{0}(\tilde{\mathbf{R}})} \delta\left(\mathbf{V}^{\prime}-\mathbf{V}^{\#}(\tilde{\mathbf{R}})\right),
$$

while $\epsilon_{K l}\left(\tilde{\mathbf{R}}_{u}, \mathbf{V}^{\prime}\right)$ is the eigenenergy of the following protonic Schrödinger equation:

$$
\hat{h}_{K}\left(\mathbf{V}^{\prime}\right) \varphi_{K l}\left(\mathbf{r}_{p} ; \tilde{\mathbf{R}}_{u}, \mathbf{V}^{\prime}\right)=\epsilon_{K l}\left(\tilde{\mathbf{R}}_{u}, \mathbf{V}^{\prime}\right) \varphi_{K l}\left(\mathbf{r}_{p} ; \tilde{\mathbf{R}}_{u}, \mathbf{V}^{\prime}\right) .
$$

The above rate expression has an interesting implication for the quantization of proton on a classical free-energy surface. To see this, let us rewrite Eq. (38) as

$$
\begin{aligned}
k_{\mathrm{ET}}= & \kappa \frac{1}{Z_{\mathrm{I}} / Z_{v}} \sum_{m} \sum_{n} \int d \tilde{\mathbf{R}}_{u} \int d \mathbf{V}^{\prime} e^{-\beta F_{\mathrm{I} m}\left(\tilde{\mathbf{R}}_{u}, \mathbf{V}^{\prime}\right)} \\
& \times\left|T_{m n}\left(\tilde{\mathbf{R}}_{u}, \mathbf{V}^{\prime}\right)\right|^{2} \delta\left[F_{\mathrm{I} m}\left(\tilde{\mathbf{R}}_{u}, \mathbf{V}^{\prime}\right)-F_{\mathrm{II} n}\left(\tilde{\mathbf{R}}_{u}, \mathbf{V}^{\prime}\right)\right],
\end{aligned}
$$

by defining a quantized free energy $F_{K l}\left(\tilde{\mathbf{R}}_{u}, \mathbf{V}^{\prime}\right)$ as follows:

$$
F_{K l}\left(\tilde{\mathbf{R}}_{u}, \mathbf{V}^{\prime}\right)=\epsilon_{K l}\left(\tilde{\mathbf{R}}_{u}, \mathbf{V}^{\prime}\right)+S_{0}^{\#}\left(\tilde{\mathbf{R}}_{u}, \mathbf{V}^{\prime}\right)
$$

This quantized free energy can be obtained equivalently by solving the following Schrödinger equation:

$$
\begin{gathered}
{\left[\hat{T}_{p}+F_{K}\left(\mathbf{R}_{u}, \mathbf{V}^{\prime}\right)\right] \varphi_{K l}\left(\mathbf{r}_{p} ; \tilde{\mathbf{R}}_{u}, \mathbf{V}^{\prime}\right)} \\
\quad=F_{K l}\left(\tilde{\mathbf{R}}_{u}, \mathbf{V}^{\prime}\right) \varphi_{K l}\left(\mathbf{r}_{p} ; \tilde{\mathbf{R}}_{u}, \mathbf{V}^{\prime}\right),
\end{gathered}
$$

where the following (classical) free energy:

$$
F_{K}\left(\mathbf{R}_{u}, \mathbf{V}^{\prime}\right)=E_{K}\left(\mathbf{R}_{u}, \mathbf{V}^{\prime}\right)+S_{0}^{\#}\left(\tilde{\mathbf{R}}_{u}, \mathbf{V}^{\prime}\right),
$$

has been used as the proton "potential." That is, within a single-point approximation like Eq. (36), the vibronic free energy $F_{K l}$ can be obtained by quantizing the proton directly on a classical free-energy surface. This observation is interesting because, in previous works employing a dielectric continuum model, PCET reactions were discussed based entirely on free-energy surfaces. For example, Cukier utilized a protonic Schrödinger equation of the form ${ }^{18-20}$

$$
\left[\hat{T}_{p}+F_{\mathrm{eq}}^{K}\left(\mathbf{R}_{u}\right)\right] \varphi_{K l}\left(\mathbf{r}_{p} ; \tilde{\mathbf{R}}_{u}\right)=F_{K l}\left(\tilde{\mathbf{R}}_{u}\right) \varphi_{K l}\left(\mathbf{r}_{p} ; \tilde{\mathbf{R}}_{u}\right),
$$

where the equilibrium solvation free energy was used as the proton potential. This choice thus neglected all the nonequilibrium solvation effects that may affect the protonic eigen- 


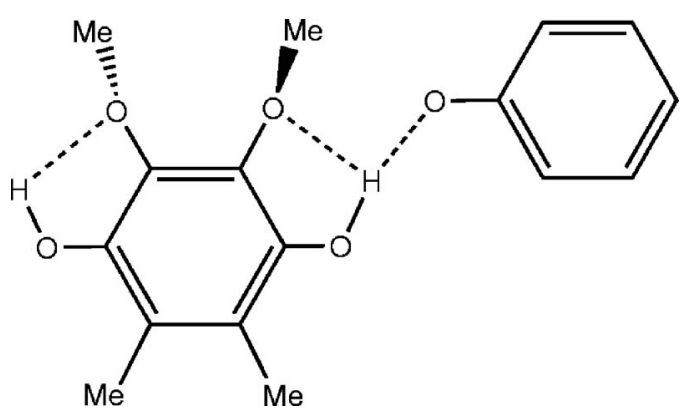

FIG. 1. Hydrogen-bonding complex of a ubiquinol analog and phenoxyl radical. Dashed lines indicate intra- and intermolecular hydrogen bonds.

states. Subsequently, Soudackov and Hammes-Schiffer developed an improved rate theory, ${ }^{21,22}$ where they introduced two scalar solvent variables, $z_{p}$ and $z_{e}$. These solvent variables represent the diabatic energy gap for single proton and single electron transfer processes, respectively, and they characterize the nonequilibrium polarization state of the dielectric. Their protonic Schrödinger equation reads qualitatively as

$$
\begin{gathered}
{\left[\hat{T}_{p}+F_{K}\left(\mathbf{R}_{u}, \mathbf{V}\left(z_{p}, z_{e}\right)\right)\right]_{\varphi_{K l}}\left(\mathbf{r}_{p} ; \tilde{\mathbf{R}}_{u}, z_{p}, z_{e}\right)} \\
\quad=F_{K l}\left(\tilde{\mathbf{R}}_{u}, z_{p}, z_{e}\right) \varphi_{K l}\left(\mathbf{r}_{p} ; \tilde{\mathbf{R}}_{u}, z_{p}, z_{e}\right),
\end{gathered}
$$

which may be regarded as a restricted solvent-space version of Eq. (43). Here, the most important solvent variables (i.e., $z_{p}$ and $z_{e}$ ) have been retained so as to simplify the description of the rate process (see the original literature for more rigorous discussions). ${ }^{21,22}$

\section{APPLICATION TO A UBIQUINOL-PHENOXYL COMPLEX IN ACETONITRILE}

\section{A. Electronic states}

We now apply the above method to the PCET reaction of a ubiquinol analog (2,3-methoxyl 5,6-methyl $p$-benzoquinol) with phenoxyl radical in acetonitrile (Fig. 1), where the isoprenoid units of natural ubiquinol are replaced by a methyl group for computational simplicity. ${ }^{47}$ As mentioned in the Introduction, our choice of this system is motivated by a recent experiment by Cape et al. ${ }^{25}$ where an interesting, inverted kinetic isotope effect (KIE) [i.e., $\Delta G^{\ddagger}(H)>\Delta G^{\ddagger}(D)$ ] has been observed for the PCET reaction of ubiquinol with a poly-pyridyl ruthenium complex in acetonitrile. A similar inverted KIE was also observed for the oxidation of ubiquinol by a tocopherol derivative (vitamin E) in ethanol. ${ }^{26}$

In the present PCET reaction, the active electron transfers from the highest doubly occupied $\pi$ orbital of ubiquinol to the singly occupied $\pi$ orbital of phenoxyl radical, while the proton transfers between the two phenolic oxygen atoms. In fact, one can think of two distinct reaction pathways, namely the classic HAT and PCET pathways, by assuming that the present reaction proceeds in a qualitatively similar manner to the phenol/phenoxyl self-exchange reaction in the gas phase (see Fig. 1 of Ref. 16). In the HAT pathway of the phenol/phenoxyl reaction, the transferring proton approaches the unpaired electron of the acceptor oxygen atom (thus inducing a three-electron, three-center process), while in the
PCET pathway the proton approaches the lone-pair electrons of the acceptor oxygen (a four-electron, three-center process). In this paper we focus only on the PCET pathway because the HAT pathway is shown to possess a much higher activation energy than the PCET one in the case of the phenol/phenoxyl complex. ${ }^{16}$

Hereafter, we assume that the electron transfer is in the nonadiabatic regime and one can make the golden rule approximation discussed in Sec. II. This assumption seems valid because the spatial overlap of $\pi$ orbitals on ubiquinol and phenoxyl radical is rather small. With this assumption we calculated the diabatic electronic wave functions of the complex using the restricted open-shell Hartree-Fock (ROHF) plus second-order Møller-Plesset perturbation (MP2) theory. The diabatic character of the wave function was maintained by preparing the ROHF wave function of a noninteracting complex with a prescribed diabatic character and then evaluating the total energy of the corresponding interacting system at the ROHF/MP2 level. The basis set used was $(9 s 5 p 1 d) /[3 s 2 p 1 d]$ for $\mathrm{C}$ and $\mathrm{O}$ atoms, and $(4 s) /[2 s]$ for $\mathrm{H}$ atoms, with additional polarization functions on hydroxyl hydrogens. ${ }^{48}$ All the calculations were performed with our modified version of the GAMESS quantum chemistry package. ${ }^{49}$

\section{B. Equilibrium solvation free-energy surfaces}

The first step for studying the title reaction is to examine the topography of equilibrium solvation free-energy surfaces as a function of solute nuclear coordinates. In this section we use the following definition of equilibrium solvation freeenergy [equivalent with Eq. (A26)]:

$$
F_{\mathrm{eq}}\left(\mathbf{R}_{u}\right)=-\beta^{-1} \ln \frac{1}{Z_{v}} \int d \mathbf{R}_{v} \exp \left\{-\beta U\left[\mathbf{R} ; \Psi_{\mathrm{eq}}\left(\mathbf{R}_{u}\right)\right]\right\},
$$

where the solvent is in equilibrium with partial charges of the solute (having a fixed geometry), while the solute wave function $\Psi_{\text {eq }}$ is calculated under the average electrostatic field from the solvent. We calculated the above free energy using the RISM-ROHF/MP2 method, ${ }^{28,29}$ where the solvent is described in terms of site-site radial distribution functions. The RISM integral equation involved was solved with the hypernetted-chain closure (HNC) relation at the solvent temperature and density of $295 \mathrm{~K}$ and $0.777 \mathrm{~g} / \mathrm{cm}^{3}$, respectively. The MM force-field parameters for $\mathrm{CH}_{3} \mathrm{CN}$ were taken from Ref. 50, while those for the solute-solvent LJ interactions were from AMBER. ${ }^{51}$ Partial charges on the solute sites were determined using the RESP method. ${ }^{30}$ Figure 2 displays the equilibrium free-energy surfaces thus obtained as a function of proton coordinate $r$ and donor-acceptor distance $R$ given by

$$
\begin{aligned}
& r=R(\mathrm{OH})-R\left(\mathrm{O}^{\prime} H\right), \\
& R=R\left(\mathrm{OO}^{\prime}\right),
\end{aligned}
$$

where $H$ denotes the transferring proton while $\mathrm{O}$ and $\mathrm{O}^{\prime}$ are the donor and acceptor oxygen atoms, respectively. The remaining solute coordinates were optimized at the RISM- 


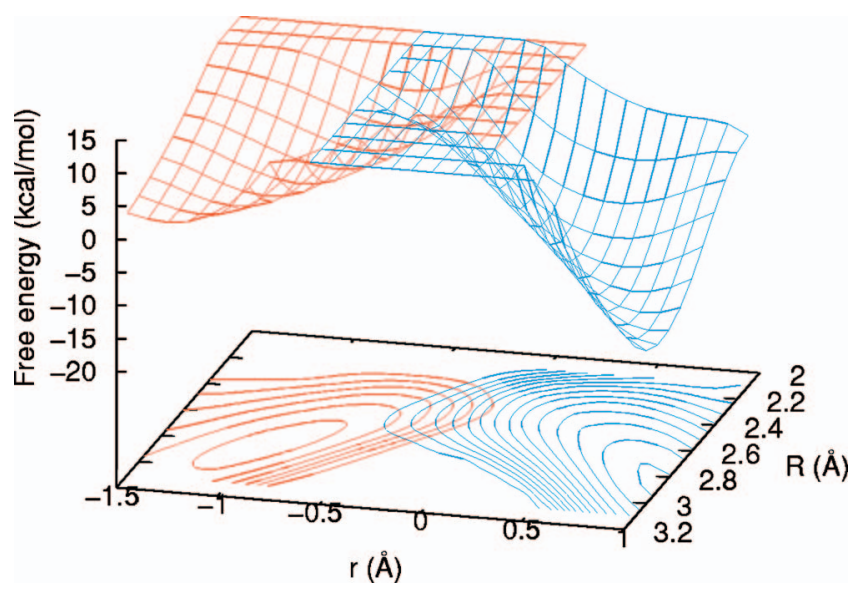

FIG. 2. (Color) Equilibrium solvation free energy defined by Eq. (47) as a function of proton coordinate $r$ and donor-acceptor distance $R$ given by Eqs. (48a) and (48b). Red and blue lines represent the reactant and product diabatic free-energy surfaces, respectively.

ROHF level. Dynamic electron correlation energy was further estimated with the MP2 method. ${ }^{52}$ The two surfaces in Fig. 2 describe the reactant and product diabatic states, denoted as state I and II, respectively. In state I the active electron resides on the ubiquinol side, while in state II it resides on the phenoxyl side. The minimum free-energy point on each surface was found to be $(r, R)$ $=(-0.976,2.801) \AA$ for state I and $(r, R)=(0.956,2.841) \AA$ for state II. This length of donor-acceptor distance indicates a weak to moderate hydrogen bond, and accordingly the equilibrium position of the proton is different by about $1 \AA$ between the two states. The corresponding solute geometry (not shown here) was qualitatively similar to that of the phenol/phenoxyl complex in the gas phase. ${ }^{16}$ The main features include: (i) two benzene rings are nearly coplanar, with methoxyl methyl groups tilted in the out-of-plane direction, and (ii) the transferring proton is doubly hydrogen-bonded to the acceptor oxygen atom of phenoxyl radical and to the oxygen atom of the $o$-methoxyl group of ubiquinol (indicated by dashed lines in Fig. 1). The reaction free energy, defined here as the difference of minimum free-energy values of state I and II, was estimated to be $-15.6 \mathrm{kcal} / \mathrm{mol}$, indicating that this reaction is strongly exothermic in acetonitrile. Though the two surfaces exhibit a crossing seam in Fig. 2, this crossing does not provide a useful estimate of the activation free energy because the solute coordinates other than $r$ and $R$ as well as the solvent distribution are optimized (or equilibrated) separately for each diabatic state and thus take different values on the crossing seam.

The dipole moment of the solute under equilibrium solvation is plotted in Fig. 3 as a function of proton coordinate $r$. For an illustrative purpose, the donor-acceptor distance $R$ was fixed at $2.6 \AA$, which is an intermediate value between $R \sim 2.4 \AA$ (around the crossing region) and $R \sim 2.8 \AA$ (the equilibrium regions) as seen in Fig. 2. Figure 3 reveals that the dipole moment for each diabatic state amounts to 12-14 debye as the proton approaches the midpoint of the donor and acceptor oxygen atoms. This large change in the dipole moment is partly due to the spatial separation of two phenyl groups, whose partial charges (in addition to those in the

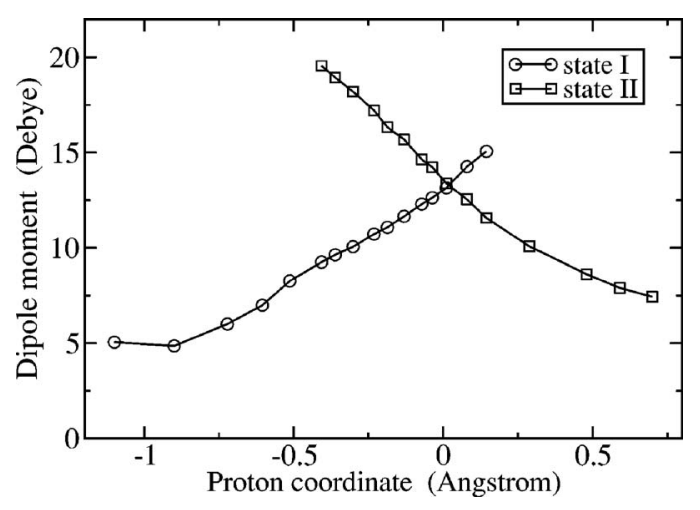

FIG. 3. Dipole moment of the complex under equilibrium solvation as a function of proton coordinate $r$. Line with circles (squares) represents the reactant (product) diabatic state. Note that the dipole moment orients from the ubiquinol side to the phenoxyl side in state I and vice versa in state II.

hydrogen-bond interface) vary as a function of proton coordinate $r$. Note that the orientation of dipole moment is antiparallel between the two states; it orients from the ubiquinol to phenoxyl side in state I and vice versa in state II. This is because the sign of partial charges (particularly on phenyl groups) are reversed between the two states, reflecting the transfer of a $\pi$-electron between the phenyl groups. This large change in the dipole moment induces a significant reorganization of solvent upon proton and electron transfer.

\section{Transition state in the combined solute-solvent space}

In order to calculate the activation free energy for electron transfer, one needs to take into account the matching condition between diabatic potential energies, namely $U_{\mathrm{I}}(\mathbf{R})=U_{\mathrm{II}}(\mathbf{R})[$ cf. Eq. (15)], which is lacking in the definition of equilibrium solvation free energy in Eq. (47). This matching condition is treated traditionally by calculating the probability density of the diabatic energy gap. As discussed in Sec. II C, an alternative approach is to express the matching condition in terms of $V$-resolved free energy, where the external potential $\mathbf{V}(\mathbf{R})$ is employed as collective solvent coordinates. The relevant situation is illustrated in Fig. 4, where the diabatic free energies, $F_{\mathrm{I}}\left(\mathbf{R}_{u}, \mathbf{V}\right)$ and $F_{\mathrm{II}}\left(\mathbf{R}_{u}, \mathbf{V}\right)$, are plotted as functions of $\mathbf{R}_{u}$ and $\mathbf{V}$ (note that multidimensional quantities, $\mathbf{R}_{u}$ and $\mathbf{V}$, are projected onto onedimensional axes). In this figure, the $\mathbf{R}_{u}$ axis specifies various solute geometries while the $\mathbf{V}$ axis specifies various solvation structures. The electron transfer proceeds by starting from the reactant (point $A$ in Fig. 4), passing through an intermediate crossing point $(X)$, and ends at the product $(B)$. In the $\mathbf{V}$ direction, $F_{\mathrm{I}}$ and $F_{\mathrm{II}}$ are multidimensional paraboloids to a very good approximation [cf. Eq. (A23)]. Dashed lines in Fig. 4 represent the equilibrium solvation paths for the individual diabatic states, where $\mathbf{V}$ is equilibrated to the solute partial charges at each $\mathbf{R}_{u}$. The equilibrium value of $\mathbf{V}$ will be denoted as $\mathbf{V}_{\mathrm{eq}}^{K}\left(\mathbf{R}_{u}\right)(K=\mathrm{I}, \mathrm{II})$. Note that these equilibrium solvation paths correspond to the equilibrium solvation free-energy surfaces plotted in Fig. 2. The vertical arrow in Fig. 4 indicates the free-energy cost for attaining nonequilibrium solvation or desolvation (i.e., to reach the crossing point $X)$. 


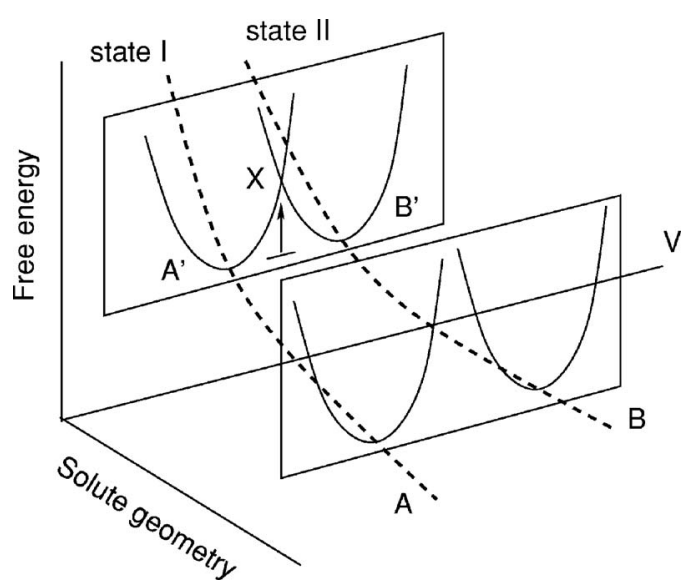

FIG. 4. Schematic illustration of the $V$-resolved free energy, $F\left(\mathbf{R}_{u}, \mathbf{V}\right)$, as a function of the solute nuclear coordinates $\mathbf{R}_{u}$ and the external potential $\mathbf{V}$. Points $A$ and $B$ represent the reactant and product configurations, $A^{\prime}$ and $B^{\prime}$ are thermally excited states under equilibrium solvation, and $X$ is the minimum free-energy crossing (MFX) point between the two diabatic freeenergy surfaces. Dashed lines represent the equilibrium solvation paths on the individual diabatic states. The vertical arrow represents the nonequilibrium solvation (or desolvation) process from the equilibrium solvation path.

Our task is then to find the minimum free-energy crossing (MFX) point (i.e., the "transition state") in the reduced $\left(\mathbf{R}_{u}, \mathbf{V}\right)$ space. However, it should be noted that such a task can be very demanding computationally unless analytical derivatives of $F_{K}\left(\mathbf{R}_{u}, \mathbf{V}\right)$ are available with respect to $\mathbf{R}_{u}$ and V. Since such a derivative is not available for the RISMROHF/MP2 method at present, we have performed a gridbased optimization that locates the MFX point approximately in a reduced-dimensional space. Specifically, we first parametrize the solute geometry in terms of three variables, $r, R$, and $s$, where $r$ and $R$ are those defined by Eqs. (48a) and (48b), while $s$ is a linear interpolation parameter that describes the solute backbone geometry as follows:

$$
q_{i}(r, R, s)=(1-s) q_{i}^{\mathrm{I}, \mathrm{opt}}(r, R)+s q_{i}^{\mathrm{II}, \mathrm{opt}}(r, R) .
$$

Here, $\left\{q_{i}\right\}(i=1, \ldots, 112)$ denotes the internal coordinates of the solute except for $(r, R)$, and $\left\{q_{i}^{K, \text { opt }}(r, R)\right\}(K=\mathrm{I}$,II $)$ are those optimized at the RISM-ROHF level at given values of $(r, R)$. We then calculated the following free energy (see Appendix A for its derivation):

$$
\begin{aligned}
F_{K}(r, R, s, \mathbf{V})= & F_{\mathrm{eq}}^{K}(r, R, s)+\Delta F_{\mathrm{elec}}^{K}(r, R, s, \mathbf{V}) \\
& +\Delta F_{\mathrm{or}}^{K}(r, R, s, \mathbf{V}),
\end{aligned}
$$

where $\Delta F_{\text {elec }}^{K}$ represents the contribution from solute electronic polarization,

$$
\Delta F_{\mathrm{elec}}^{K}(r, R, s, \mathbf{V})=\frac{1}{2}\left(\mathbf{V}-\mathbf{V}_{\mathrm{eq}}^{K}\right) \cdot \mathbf{K}_{\mathrm{eq}}^{K} \cdot\left(\mathbf{V}-\mathbf{V}_{\mathrm{eq}}^{K}\right),
$$

while $\Delta F_{\text {or }}^{K}$ is the contribution from solvent orientational polarization,

$$
\Delta F_{\mathrm{or}}^{K}(r, R, s, \mathbf{V})=\frac{1}{2 \beta}\left(\mathbf{V}-\mathbf{V}_{\mathrm{eq}}^{K}\right) \cdot\left[\mathbf{C}_{\mathrm{eq}}^{K}\right]^{-1} \cdot\left(\mathbf{V}-\mathbf{V}_{\mathrm{eq}}^{K}\right) .
$$

$\mathbf{K}_{\text {eq }}^{K}$ in Eq. (51) is the charge-response kernel (CRK), ${ }^{31-33}$ which was calculated by three-point differentiation of solute partial charges with respect to the external potential, while
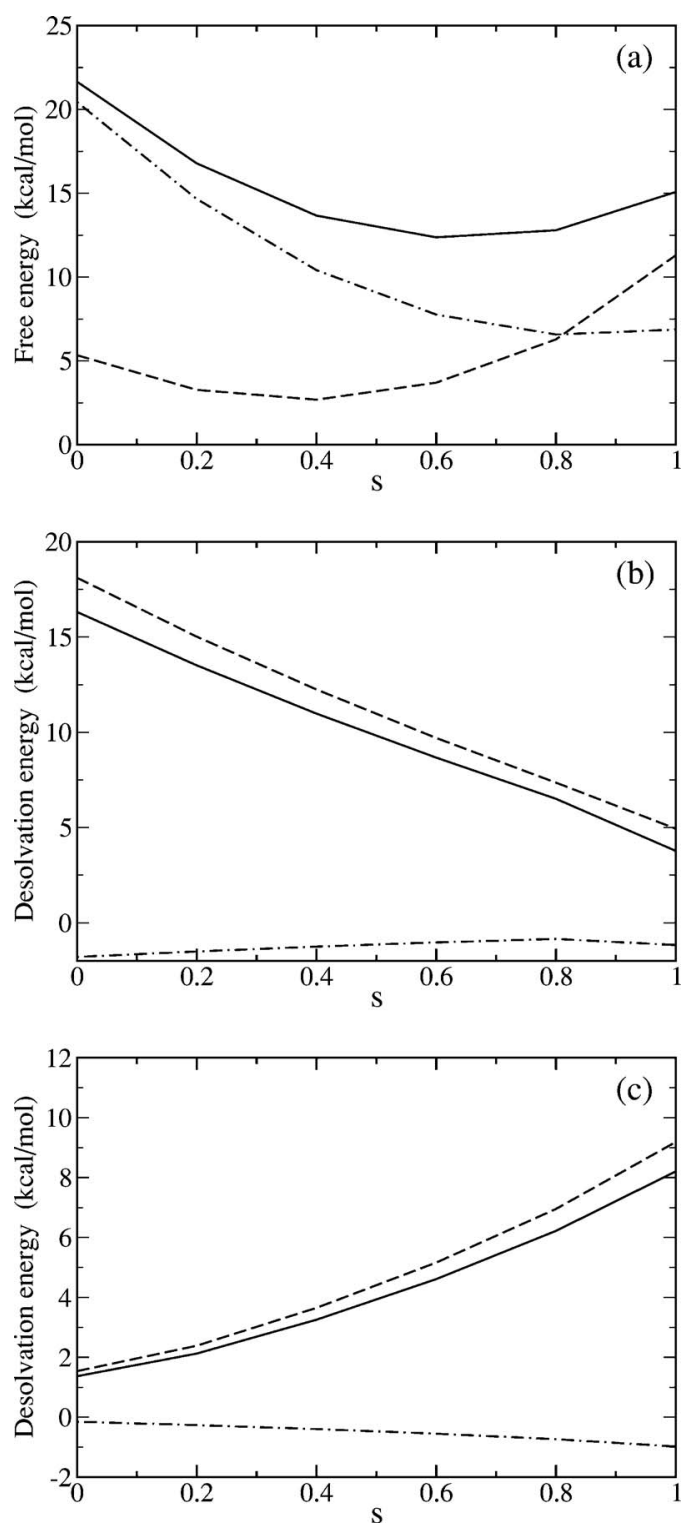

FIG. 5. (a) $F_{X}(r, R, s)$ defined by Eq. (53) as a function of $s$ at $(r, R)$ $=(-0.33,2.4) \AA$ (solid line), where $s$ is a linear interpolation parameter of the solute backbone geometry. Dashed and dash-dotted lines represent the equilibrium solvation free energy for the reactant and product diabatic states, $F_{\text {eq }}^{\mathrm{I}}(r, R, s)$ and $F_{\text {eq }}^{\mathrm{II}}(r, R, s)$, respectively. (b) Decomposition of the desolvation energy, $F_{X}(r, R, s)-F_{\mathrm{eq}}^{\mathrm{I}}(r, R, s)$, for the reactant diabatic state: Dash-dotted, dashed, and solid lines represent $\Delta F_{\text {elec }}^{\mathrm{I}}(r, R, s), \Delta F_{\text {or }}^{\mathrm{I}}(r, R, s)$, and their sum, respectively. (c) The same as (b) but for the product diabatic state $(K=\mathrm{II})$

$\mathbf{C}_{\mathrm{eq}}^{K}$ in Eq. (52) is the covariance matrix of $\mathbf{V}(\mathbf{R})$ that was calculated analytically by solving the coupled perturbed RISM equation. ${ }^{8,53}$ Note that all of the $\mathbf{V}_{\mathrm{eq}}^{K}, \mathbf{K}_{\mathrm{eq}}^{K}$, and $\mathbf{C}_{\mathrm{eq}}^{K}$ are functions of $(r, R, s)$. We then minimized the above free energy with respect to $\mathbf{V}$ within the crossing seam, i.e.,

$$
\begin{aligned}
F_{X}(r, R, s)= & \min _{\mathbf{V}, \lambda}\left\{F_{\mathrm{I}}(r, R, s, \mathbf{V})+\lambda\left[F_{\mathrm{I}}(r, R, s, \mathbf{V})\right.\right. \\
& \left.\left.-F_{\mathrm{II}}(r, R, s, \mathbf{V})\right]\right\},
\end{aligned}
$$

where $\lambda$ is the Lagrange multiplier. We denote the value of $\mathbf{V}$ that minimizes the right-hand side of Eq. (53) as $\mathbf{V}_{X}$ $=\mathbf{V}_{X}(r, R, s)$ and refer to $\left(r, R, s, \mathbf{V}_{X}\right)$ as a local crossing point at a given solute geometry. Figure 5 displays the 


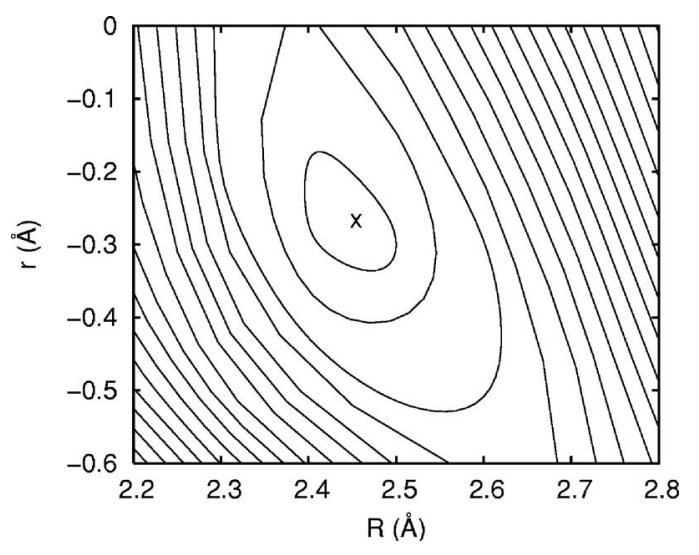

FIG. 6. Plot of $\min _{s} F_{X}(r, R, s)$ as a function of $r$ and $R$. The cross symbol indicates the location of the minimum free-energy crossing point. Contours are plotted with a spacing of $1 \mathrm{kcal} / \mathrm{mol}$, starting from $12 \mathrm{kcal} / \mathrm{mol}$.

section of $F_{X}(r, R, s)$ as a function of $s$ at $(r, R)$ $=(-0.33,2.4) \AA$ together with the equilibrium solvation free energies, $F_{\mathrm{eq}}^{\mathrm{I}}(r, R, s)$ and $F_{\mathrm{eq}}^{\mathrm{II}}(r, R, s)$. This figure reveals that $F_{X}$ relaxes by as much as $7 \mathrm{kcal} / \mathrm{mol}$ when one varies $s$ from 0 to 0.6 , indicating that such a geometrical change can be important in accurately estimating the activation energy. Note that $F_{X}$ is always greater than both $F_{\text {eq }}^{\mathrm{I}}$ and $F_{\text {eq }}^{\mathrm{II}}$ due to the presence of desolvation energy (indicated by the vertical arrow in Fig. 4). Figures 5(b) and 5(c) plot the desolvation energy $F_{X}-F_{\text {eq }}^{K}$ and its solute and solvent components, $\Delta F_{\text {elec }}^{K}\left(r, R, s, \mathbf{V}_{X}\right)$ and $\Delta F_{\text {or }}^{K}\left(r, R, s, \mathbf{V}_{X}\right)$. These figures indicate that the distortion of solute wave function from $\Psi\left(\mathbf{V}_{\mathrm{eq}}^{K}\right)$ to $\Psi\left(\mathbf{V}_{X}\right)$ is of minor importance compared to the solvent orientational reorganization. As the last step of the transitionstate search, Fig. 6 displays $\min _{s} F_{X}(r, R, s)$ as a function of $(r, R)$, from which the global MFX point was identified to be $\left(r^{\ddagger}, R^{\ddagger}, s^{\ddagger}\right)=(-0.265 \AA, 2.447 \AA, 0.606)$, and the associated activation energy [defined as $\Delta F^{*}=F_{X}\left(\mathbf{R}_{u}^{\ddagger}\right)-F_{\text {eq }}^{\mathrm{I}}\left(\mathbf{R}_{u, \text { eq }}^{\mathrm{I}}\right)$ ] was estimated to be $11.6 \mathrm{kcal} / \mathrm{mol}$. This activation energy can be further divided into equilibrium and nonequilibrium solvation components, namely, $\Delta F^{*}=\Delta F_{\text {eq }}^{\ddagger}+\Delta F_{\text {neq }}^{\dagger}$, where $\Delta F_{\text {eq }}^{*}$ is the free-energy difference along the equilibrium solvation path in the $\left(\mathbf{R}_{u}, \mathbf{V}\right)$ space $\left(A \rightarrow A^{\prime}\right.$ in Fig. 4$)$, while $\Delta F_{\text {neq }}^{\ddagger}$ is the desolvation energy at the transition state $\mathbf{R}_{u}^{\ddagger}\left(A^{\prime} \rightarrow X\right.$ in Fig. 4). The result is

$$
\begin{aligned}
& \Delta F_{\text {eq }}^{\ddagger}=F_{\text {eq }}^{\mathrm{I}}\left(\mathbf{R}_{u}^{\ddagger}\right)-F_{\text {eq }}^{\mathrm{I}}\left(\mathbf{R}_{u, \text { eq }}^{\mathrm{I}}\right)=5.1 \mathrm{kcal} / \mathrm{mol}, \\
& \Delta F_{\text {neq }}^{\ddagger}=F_{\mathrm{I}}\left(\mathbf{R}_{u}^{\ddagger}, \mathbf{V}^{\ddagger}\right)-F_{\text {eq }}^{\mathrm{I}}\left(\mathbf{R}_{u}^{\ddagger}\right)=6.5 \mathrm{kcal} / \mathrm{mol},
\end{aligned}
$$

where $\mathbf{V}^{\ddagger}$ denotes the external potential at the global MFX point. The equilibrium and nonequilibrium solvation thus makes similar contribution to the total activation energy for the present system. Furthermore, the components $\Delta F_{\text {elec }}^{\ddagger}$ and $\Delta F_{\text {or }}^{\sharp}$ were estimated to be -0.8 and $7.3 \mathrm{kcal} / \mathrm{mol}$, respectively, again indicating a relatively minor importance of the former to the total activation energy.

To understand the nonequilibrium solvation more intuitively, Fig. 7 visualizes the electrostatic potential produced by the solvent upon solute sites. Under the equilibrium solvation in state I [panel (a)], the external potential is positive on the ubiquinol side (in an average sense) and negative on the phenoxyl side. This is because the phenyl carbon atoms
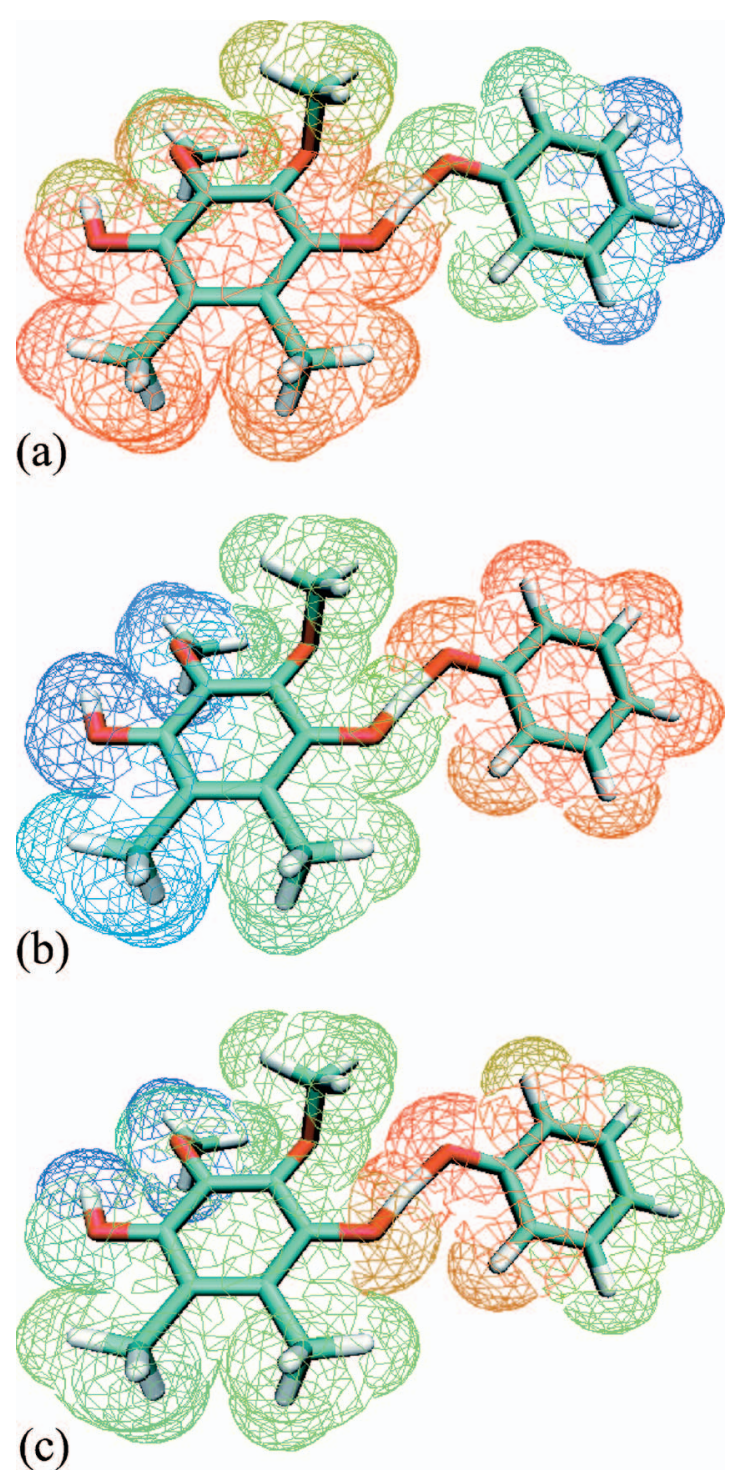

FIG. 7. (Color) Solvent electrostatic potential $\mathbf{V}$ acting on solute atomic sites. Panels (a), (b), and (c) depict $\mathbf{V}_{\text {eq }}^{\mathrm{I}}\left(\mathbf{R}_{u}^{\ddagger}\right), \mathbf{V}_{\mathrm{eq}}^{\mathrm{II}}\left(\mathbf{R}_{u}^{\ddagger}\right)$, and $\mathbf{V}^{\ddagger}$, respectively. Red, blue, and green lines around the individual atoms indicate positive, negative, and intermediate values of the solvent electrostatic potential, respectively.

of ubiquinol are negatively charged and the solvent stabilizes them with positive values of the external potential. Under the equilibrium solvation in state II [panel (b)], the external potential exhibits a qualitatively inverted character from panel (a). This is because the ubiquinol phenyl group becomes more positively charged due to the electron transfer from the ubiquinol to the phenoxyl side. At the transition state [panel (c)], the external potential exhibits an intermediate character between panels (a) and (b), as expected, which suggests that neither the ubiquinol nor phenoxyl atoms are stabilized more strongly by the solvent. This unbiased solvation facilitates the matching of diabatic potential energies, which is required for electron transfer events to occur (within the classical golden rule approximation).

\section{Reaction rates and kinetic isotope effects}

In the previous sections we have assumed the classical treatment of the proton, and here we wish to quantize it in 


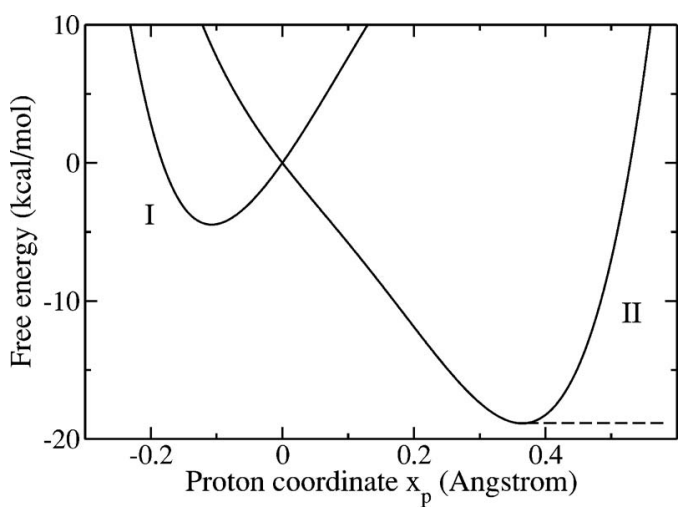

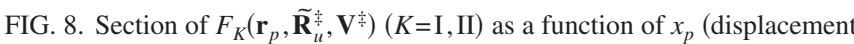
of proton along the line connecting the donor-acceptor oxygens). The origin of the abscissa and ordinate is chosen at the (classical) minimum free-energy crossing point. Dashed line represents the modification of the product potential to a dissociative one [cf. Eq. (59)].

order to discuss kinetic isotope effects (KIE). For this purpose, we employ the simplest version of the rate expressions derived in Sec. II D [cf. Eq. (41)],

$$
\begin{aligned}
k_{\mathrm{ET}}= & \kappa \frac{1}{Z_{I}} \sum_{m} \sum_{n} \int d \mathbf{X} e^{-\beta F_{\mathrm{I} m}(\mathbf{X})}\left|T_{m n}(\mathbf{X})\right|^{2} \\
& \times \delta\left[F_{\mathrm{I} m}(\mathbf{X})-F_{\mathrm{II} n}(\mathbf{X})\right],
\end{aligned}
$$

with $Z_{I}=\Sigma_{m} \int d \mathbf{X} \exp \left[-\beta F_{\mathrm{I} m}(\mathbf{X})\right]$, where $\mathbf{X}=\left(\widetilde{\mathbf{R}}_{u}, \mathbf{V}\right)$ is the classical coordinates of the system excluding the proton and $F_{\mathrm{I} m}(\mathbf{X})$ is the eigenenergy of the following protonic Schrödinger equation:

$$
\left[\hat{T}_{p}+F_{\mathrm{I}}\left(\mathbf{R}_{u}, \mathbf{V}\right)\right]_{\varphi_{\mathrm{I} m}}\left(\mathbf{r}_{p} ; \mathbf{X}\right)=F_{\mathrm{I} m}(\mathbf{X}) \varphi_{\mathrm{I} m}\left(\mathbf{r}_{p} ; \mathbf{X}\right) .
$$

Note that the classical free energy $F_{\mathrm{I}}\left(\mathbf{R}_{u}, \mathbf{V}\right)$ is used as the proton "potential," which can be justified under the probepoint approximation discussed above. Figure 8 displays the section of $F_{\mathrm{I}}\left(\mathbf{r}_{p}, \mathbf{X}^{\ddagger}\right)$ and $F_{\mathrm{II}}\left(\mathbf{r}_{p}, \mathbf{X}^{\ddagger}\right)$, with $\mathbf{X}^{\ddagger}=\left(\mathbf{R}_{u}^{\ddagger}, \mathbf{V}^{\ddagger}\right)$ as a function of $x_{p}$ (here, the $x$ axis is chosen in the direction from the donor to acceptor oxygen atoms). ${ }^{54}$ Here, we evaluate Eq. (55) very approximately by using only the information about the reactant and transition-state configurations, thus similar in spirit to classical transition state theory (TST). Specifically, we extract the integrand of Eq. (55) to define the following relative reaction rate $\tilde{k}$ :

$$
k_{\mathrm{ET}} \propto \tilde{k} \equiv \frac{\Sigma_{m} \exp \left[-\beta F_{\mathrm{I} m}\left(\mathbf{X}^{\ddagger}\right)\right] D_{m}^{\ddagger}}{\sum_{m} \exp \left[-\beta F_{\mathrm{I} m}\left(\mathbf{X}_{\mathrm{eq}}^{\mathrm{I}}\right)\right]},
$$

where $D_{m}^{\ddagger}$ is a type of Franck-Condon weighted density,

$$
D_{m}^{\ddagger}=\sum_{n}\left|T_{m n}\left(\mathbf{X}^{\ddagger}\right)\right|^{2} \delta\left[F_{\mathrm{I} m}\left(\mathbf{X}^{\ddagger}\right)-F_{\mathrm{II} n}\left(\mathbf{X}^{\ddagger}\right)\right] .
$$

Note that only the factors that most likely impact the KIE have been retained, and that the possibly important variation in the donor-acceptor distance has also been neglected. This approximation is motivated by the expectation that the integrand in Eq. (55) may take the maximum value at around the classical MFX point (after including the summation over $m$ and $n$ into the integrand). Our idea is simply to take the ratio of $\tilde{k}$ in Eq. (57) for proton $(H)$ and deuteron
TABLE I. Quantized free energies (in $\mathrm{kcal} / \mathrm{mol}$ ) measured from the bottom of the proton potential, i.e., $\Delta \epsilon_{m}(\mathbf{X})=F_{\mathrm{I} m}(\mathbf{X})-\min F_{\mathrm{I}}\left(\mathbf{r}_{p}, \mathbf{X}\right)$ with $\mathbf{X}=\mathbf{X}_{\mathrm{eq}}^{\mathrm{I}}$ (reactant state) or $\mathbf{X}=\mathbf{X}^{\ddagger}$ (transition state). $D_{m}^{\ddagger}$ is the Franck-Condon weighted density by Eq. (58). Values of $D_{m}^{\ddagger}$ are scaled such that $D_{m}^{\ddagger}(m=0)$ becomes unity for the $H$ transfer.

\begin{tabular}{lrrr}
\hline \hline$m$ & $\Delta \boldsymbol{\epsilon}_{m}\left(\mathbf{X}_{\mathrm{eq}}^{\mathrm{I}}\right)$ & $\Delta \boldsymbol{\epsilon}_{m}\left(\mathbf{X}^{\ddagger}\right)$ & $D_{m}^{\ddagger}$ \\
\hline & \multicolumn{4}{c}{$H$ transfer } \\
1 & 7.8 & 8.8 & 1.00 \\
2 & 9.9 & 11.6 & 0.71 \\
3 & 11.7 & 12.8 & 1.30 \\
4 & 12.3 & 14.6 & 0.57 \\
& 13.7 & 15.7 & 1.20 \\
0 & & $D$ transfer & \\
1 & 5.5 & 6.2 & 0.40 \\
2 & 6.9 & 8.2 & 0.29 \\
3 & 8.2 & 9.1 & 0.88 \\
4 & 8.5 & 10.3 & 0.26 \\
\hline \hline
\end{tabular}

(D) transfer in order to estimate the KIE. However, this simple approach has a formal difficulty that $D_{m}^{\ddagger}$ vanishes (if literally evaluated) because of the discrete nature of $F_{\mathrm{I} m}$ and $F_{\text {II } n}$ in the delta function. We thus modify the above definition of $D_{m}^{\ddagger}$ slightly by first writing it in a time-dependent form,

$$
\begin{aligned}
D_{m}^{\ddagger}= & \int \frac{d t}{2 \pi \hbar} e^{i F_{\mathrm{I} m}\left(\mathbf{x}^{\ddagger}\right) t / \hbar} \\
& \times\left\langle\varphi_{\mathrm{I} m}\left(\mathbf{r}_{p} ; \mathbf{X}^{\ddagger}\right)\left|e^{-i \hat{h}_{\mathrm{II}}\left(\mathbf{X}^{\ddagger}\right) t / \hbar}\right| \varphi_{\mathrm{I} m}\left(\mathbf{r}_{p} ; \mathbf{X}^{\ddagger}\right)\right\rangle,
\end{aligned}
$$

with $\hat{h}_{\mathrm{II}}\left(\mathbf{X}^{\ddagger}\right)=\hat{T}_{p}+F_{\mathrm{II}}\left(\mathbf{r}_{p}, \mathbf{X}^{\ddagger}\right)$ and changing the shape of $F_{\text {II }}\left(\mathbf{r}_{p}, \mathbf{X}^{\ddagger}\right)$ artificially to a dissociative one (shown by the dashed line in Fig. 8). This modification seems reasonable because the product potential is much deeper than the reactant one due the exothermicity of the reaction, and thus the density of product vibrational states is rather large in the energy region near the (classical) MFX point.

Table I summarizes the quantized free energies measured from the bottom of the diabatic free-energy surface at $\mathbf{X}$ $=\mathbf{X}_{\mathrm{eq}}^{\mathrm{I}}$ or $\mathbf{X}^{\ddagger}$ (thus physically meaning the proton vibrational energy) and $D_{m}^{\ddagger}$ calculated with Eq. (59). Table I shows that the vibrational ground-state energy at the transition state is close to that of the classical MFX point, indicating that the corresponding wave function has a sufficient overlap with the product wave functions in the same energy region. This is verified by the fact that $D_{m}^{\ddagger}$ depends rather weakly on the state index $m$. The fluctuations in the factor $D_{m}^{\ddagger}$ are due to different vibrational patterns of the reactant wave function; $D_{m}^{\ddagger}$ increases when the proton is more excited along the direction from the donor to acceptor oxygen atoms, and remains the same or even decreases when the vibration is along an orthogonal direction (e.g., out of the phenyl plane). Note that the proton will be vibrationally excited when transferring from state I to state II, because the present reaction is strongly exothermic and the proton potential of state II is much lower in energy than that of state I as shown in Fig. 8. It is also interesting to note that the zero-point energy (ZPE) at the transition state, $8.8 \mathrm{kcal} / \mathrm{mol}$, is slightly greater than that in the reactant configuration, $7.8 \mathrm{kcal} / \mathrm{mol}$. The stiffness 


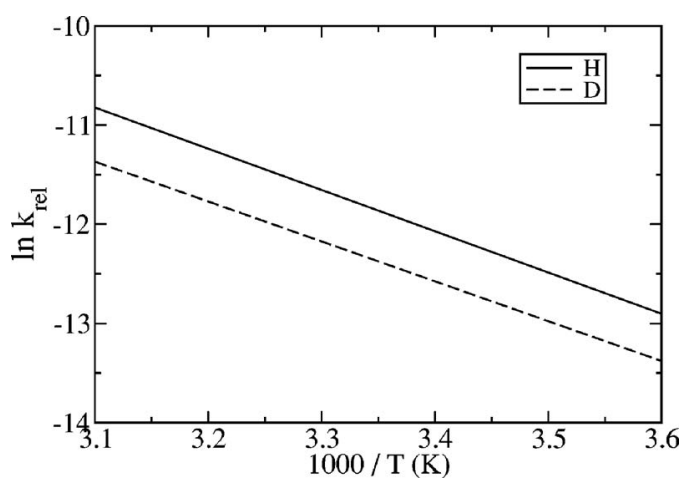

FIG. 9. Arrhenius plot of the relative reaction rate $\tilde{k}$ in Eq. (57) for the proton $(H)$ and deuteron $(D)$ transfer.

of the proton potential can be compared via normal-mode frequencies of the proton at the transition state $\mathbf{X}^{\ddagger}$ $(1006,1473,3846) \mathrm{cm}^{-1}$, and those at the reactant state $\mathbf{X}_{\mathrm{eq}}^{\mathrm{I}}$ $(726,1201,3577) \mathrm{cm}^{-1}$; i.e., the proton potential is stiffer at the transition state than at the reactant state.

By substituting the data in Table I and the classical activation energy $\Delta F^{*}=11.6 \mathrm{kcal} / \mathrm{mol}$ into Eq. (57), we obtain the Arrhenius plot of relative reaction rate $\tilde{k}$ in Fig. 9. An important observation here is that the slopes of the two curves are almost the same, suggesting that the apparent activation energies $\Delta G$ for the $H$ and $D$ transfer are very close, i.e., $\Delta G(H) \simeq \Delta G(D)$. This is in contrast with electronically adiabatic hydrogen transfer reactions in the thermally activated regime (i.e., not dominated by tunneling), where $\Delta G(H)<\Delta G(D)$ is expected from the classical transitionstate theory rate expression with zero-point energy corrections for the transferring hydrogen. ${ }^{55}$ In the present case, the similar values of $\Delta G$ for $H$ and $D$ transfer are due to the cancellation of two factors: (i) the slightly larger (ZPEcorrected) activation energy for the $H$ transfer, $11.6+8.8$ $-7.8=12.6 \mathrm{kcal} / \mathrm{mol}$, than that for the $D$ transfer, $12.3 \mathrm{kcal} /$ mol, where there is no missing degree of freedom in defining the ZPE, and (ii) the slightly larger value of $D_{m}^{\ddagger}$ for the $H$ transfer due to greater spreading of the wave function. Thus, the mechanism for determining the apparent activation energy is somewhat different from that of the electronically adiabatic hydrogen transfer reactions. The $\mathrm{KIE}$ at $23^{\circ} \mathrm{C}$ was estimated to be 1.7 by simply taking the ratio of $\tilde{k}$ in Eq. (57) for $H$ and $D$ transfer. This value of KIE is similar to the experimentally observed value of 1.8 for the PCET reaction of ubiquinol with a poly-pyridyl ruthenium complex in acetonitrile, ${ }^{25}$ though the inverted activation energy reported in previous experiments ${ }^{25,26}$ was not observed in the present work. We emphasize here that the present estimate of KIE would increase somewhat if we take into account the variation in the donor-acceptor distance around the transition state. This is because the proton can tunnel to the acceptor group before the classical coordinates $\mathbf{X}$ reaches its transition-state value $\mathbf{X}^{\ddagger}$. The impact of this corner-cutting effect depends on the balance between the variation in the Franck-Condon factor and the free-energy cost for decreasing the donor-acceptor separation. ${ }^{23}$ Nevertheless, we expect that the improved estimate of KIE would remain moderate due to the absence of any steric hindrance that precludes the approach of donor-acceptor groups. This is in contrast with the ubiquinol oxidation by a derivative of $\alpha$-tocopherol (vitamin E), where the KIE amounts to 20 because of the protection of the acceptor oxygen atom by bulky isopropyl groups. ${ }^{26}$ Large KIE values were also observed for PCET reactions in protein, where the protein environment itself prevents the approach of donor-acceptor groups. ${ }^{23}$ In the present system, there is no such steric hindrance and the donor-acceptor oxygens can establish a close contact to make a hydrogen-bond interface.

In addition to the steric hindrance effects mentioned above, the exothermicity of PCET reactions may also play a role in determining the value of KIE. To see this, let us compare the present ubiquinol/phenoxyl reaction, which is exothermic as seen in Figs. 2 and 8, with the self-exchange reaction of a phenol/phenoxyl complex in the gas phase (a thermo-neutral reaction). ${ }^{17}$ In the phenol/phenoxyl case, the Franck-Condon factor (or the vibronic coupling in the nonadiabatic ET limit) was shown to be different for $H$ and D transfer by a factor of 14 at the donor-acceptor separation of $2.4 \AA$. This is in contrast with the present result in Table I, which shows that the $H / D$ ratio of the Franck-Condon factor is on the order of unity for the ubiquinol/phenoxyl complex. Apart from several approximations made in obtaining Table I, the smaller ratio of the Franck-Condon factor for the latter case may be partly due to the strong exothermicity of the reaction. Specifically, the diabatic proton potentials of the phenol/phenoxyl system is symmetric with respect to the proton coordinate and possess a high activation barrier of about $20 \mathrm{kcal} / \mathrm{mol}$ (see Fig. 3 of Ref. 17). As a result, the Franck-Condon factor is governed by the small tail of the proton wave functions. On the other hand, in the present ubiquinol/phenoxyl system, the zero-point energy level in the reactant proton potential equals roughly the crossing-point energy of the two proton potential curves (Fig. 8), which implies that the vibrational excited-state wave functions in the product potential may exhibit a relatively large overlap with the vibrational ground-state wave function in the reactant potential. To obtain some numerical insight into this observation, we have recalculated the $D_{m}^{\ddagger}$ factor in Eq. (58) by artificially shifting the product potential curve (labeled as II in Fig. 8) vertically upward in energy by 5 and $10 \mathrm{kcal} / \mathrm{mol}$ in order to see the effect of exothermicity. The resulting value of KIE was found to be 4.9 and 13.6, respectively, thus increasing with decreasing exothermicity. Hence, in addition to steric hindrance effects between donor-acceptor groups, the free energetics of reactant and product diabatic states may also be important in determining the value of KIE.

\section{CONCLUDING REMARKS}

In this paper we have explored the use of solvent electrostatic potentials $V$ as collective solvent variables, and demonstrated how standard rate expressions for nonadiabatic electron transfer can be expressed in terms of $R$ and $V$ (here, $R$ is the solute nuclear geometry). This approach is based on the observation that $R$ and $V$ are the only external parameters that appear in the electronic Schrödinger equation of the sol- 
ute. By classifying the Cartesian coordinates of the solvent according to the value of $V$, we have naturally arrived at the definition of $V$-resolved free energy, $F(R, V)$. By construction, this $V$-resolved free energy separates neatly into the solute electronic energy $E(R, V)$ and the solvent statistical self-energy $S_{0}(R, V)$, as given by Eq. (14). This neat separation of $F(R, V)$ into electronic and statistical contributions may potentially lead to a computational saving in the number of necessary quantum-chemical calculations for the solute. This is because the present method performs in effect only a single quantum-chemical calculation of the solute for a group of solvent configurations having the same value of $V$, in contrast with a direct $\mathrm{QM} / \mathrm{MM}$ sampling in Cartesian space where quantum-chemical calculations of the solute and statistical-mechanical sampling of the solvent are inherently entangled.

We have applied the resulting scheme to the PCET reaction of a ubiquinol complex in solution and evaluated the KIE approximately using a TST-like approximation. This TST-like approximation, however, was rather crude and also neglected the important variation in the donor-acceptor distance. It is thus important to develop a more accurate approximation that does not require too much computational effort. A promising way for this direction is to introduce a reaction path in the $(R, V)$ space for each diabatic state, and limit the free-energy calculation on that reaction path, as commonly performed in gas-phase quantum chemistry. This approach requires the derivative of $F(R, V)$ with respect to $R$ and $V$, and in that respect an analytical derivative algorithm is most desirable. Free-energy derivatives are also useful in directly locating the minimum free-energy crossing point with fewer intermediate points (recall that we have used a very costly, grid-based method for the transition-state search). Future study will focus on those technical issues for more direct calculations.

\section{ACKNOWLEDGMENT}

This work was supported by the Grant-in-Aid for Scientific Research from the Ministry of Education and Science, Japan.

\section{APPENDIX A: APPROXIMATE CALCULATION OF V-RESOLVED FREE ENERGY}

\section{Uncharged reference}

The $V$-resolved free energy $F\left(\mathbf{R}_{u}, \mathbf{V}^{\prime}\right)$ can be separated into the solute electronic energy $E$ and the solvent selfenergy $S_{0}$, where calculating the former is a task of quantum chemistry while that of the latter is a purely classical, statistical-mechanical problem for the solvent. Here, we consider a simple computational scheme for the latter based on the Gaussian fluctuation model for the solvent. ${ }^{1}$ First, we rewrite Eq. (13) as

$$
e^{-\beta S_{0}\left(\mathbf{R}_{u}, \mathbf{V}^{\prime}\right)}=e^{-\beta \Delta \mu_{0}} P_{0}\left(\mathbf{V}^{\prime}\right),
$$

where $\Delta \mu_{0}=\Delta \mu_{0}\left(\mathbf{R}_{u}\right)$ is the solvation free energy of a fictitious uncharged solute, ${ }^{56}$

$$
e^{-\beta \Delta \mu_{0}}=\frac{1}{Z_{v}} \int d \mathbf{R}_{v} e^{-\beta U_{0}(\mathbf{R})},
$$

while $P_{0}\left(\mathbf{V}^{\prime}\right)$ is the probability for the solvent to produce the electrostatic potential $\mathbf{V}^{\prime}$,

$$
P_{0}\left(\mathbf{V}^{\prime}\right)=\left\langle\delta\left(\mathbf{V}^{\prime}-\mathbf{V}(\mathbf{R})\right)\right\rangle_{0},
$$

where the average $\langle\cdots\rangle_{0}$ is defined by

$$
\langle\cdots\rangle_{0}=\frac{\int d \mathbf{R}_{v} e^{-\beta U_{0}(\mathbf{R})(\cdots)}}{\int d \mathbf{R}_{v} e^{-\beta U_{0}(\mathbf{R})}} .
$$

The calculation of $P_{0}\left(\mathbf{V}^{\prime}\right)$ is somewhat tricky because $\mathbf{V}^{\prime}$ is a multidimensional quantity and the usual histogram method may fail to ensure sufficient statistics. To avoid this, we invoke a Gaussian fluctuation model for $\mathbf{V}^{\prime}$ given by ${ }^{1}$

$$
\begin{aligned}
P_{0}\left(\mathbf{V}^{\prime}\right) \simeq & {\left[\frac{\operatorname{det} \mathbf{C}_{0}^{-1}}{(2 \pi)^{N}}\right]^{1 / 2} } \\
& \times \exp \left\{-\frac{1}{2}\left(\mathbf{V}^{\prime}-\langle\mathbf{V}\rangle_{0}\right) \cdot \mathbf{C}_{0}^{-1} \cdot\left(\mathbf{V}^{\prime}-\langle\mathbf{V}\rangle_{0}\right)\right\},
\end{aligned}
$$

where $\langle\mathbf{V}\rangle_{0}$ and $\mathbf{C}_{0}$ are the mean and the covariance matrix of $\mathbf{V}(\mathbf{R})$,

$$
\begin{aligned}
& \langle\mathbf{V}\rangle_{0}=\langle\mathbf{V}(\mathbf{R})\rangle_{0}, \\
& \mathbf{C}_{0}=\left\langle\left[\mathbf{V}(\mathbf{R})-\langle\mathbf{V}\rangle_{0}\right]\left[\mathbf{V}(\mathbf{R})-\langle\mathbf{V}\rangle_{0}\right]^{T}\right\rangle_{0}
\end{aligned}
$$

(the superscript $T$ denotes a vector transpose). Inserting the above expressions into Eq. (A1), one obtains the following expression for $F\left(\mathbf{R}_{u}, \mathbf{V}^{\prime}\right)$ (with an additional approximation that $\left.\langle\mathbf{V}\rangle_{0} \simeq 0\right):^{57}$

$$
\begin{aligned}
F\left(\mathbf{R}_{u}, \mathbf{V}^{\prime}\right)= & \left\langle\Psi\left(\mathbf{V}^{\prime}\right)\left|\hat{H}_{0}\right| \Psi\left(\mathbf{V}^{\prime}\right)\right\rangle+\mathbf{Q}\left[\Psi\left(\mathbf{V}^{\prime}\right)\right] \cdot \mathbf{V}^{\prime} \\
& +\frac{1}{2 \beta} \mathbf{V}^{\prime} \cdot \mathbf{C}_{0}^{-1} \cdot \mathbf{V}^{\prime}+\Delta \mu_{0}+\delta F\left(\mathbf{C}_{0}\right),
\end{aligned}
$$

with

$$
\delta F(\mathbf{C})=-\beta^{-1} \ln \left[\frac{\operatorname{det} \mathbf{C}^{-1}}{(2 \pi)^{N}}\right]^{1 / 2} .
$$

It is interesting to note the similarity of Eq. (A8) with a nonequilibrium free-energy functional used in dielectric continuum theory, ${ }^{9-13}$

$$
\begin{aligned}
F[\Psi, \mathbf{P}]= & \left\langle\Psi\left|\hat{H}_{0}\right| \Psi\right\rangle+\sum_{\alpha} Q_{\alpha}[\Psi] \int d \mathbf{x} \frac{-\nabla \cdot \mathbf{P}(\mathbf{x})}{\left|\mathbf{R}_{u, \alpha}-\mathbf{x}\right|} \\
& +\frac{1}{2 \chi} \int d \mathbf{x P}(\mathbf{x})^{2}+\frac{1}{2} \int d \mathbf{x} \int d \mathbf{x}^{\prime} \\
& \times \frac{[-\nabla \cdot \mathbf{P}(\mathbf{x})]\left[-\nabla^{\prime} \cdot \mathbf{P}\left(\mathbf{x}^{\prime}\right)\right]}{\left|\mathbf{x}-\mathbf{x}^{\prime}\right|}
\end{aligned}
$$

where $\mathbf{P}(\mathbf{x})$ is the (inertial) polarization field of the dielectric and $\chi$ is an appropriate constant. The last two terms in Eq. (A10) represent the solvent self-energy, which are physically equivalent to the quadratic term in $\mathbf{V}^{\prime}$ in Eq. (A8) because they both represent the free-energy cost for thermal fluctuations in the solvent around an uncharged solute. 


\section{Self-consistent-field reference}

While in the above scheme one samples the solvent around a fictitious uncharged solute, one can also develop a scheme that samples the solvent around an arbitrarily charged solute. To this end, we rewrite Eq. (13) as

$$
\begin{aligned}
e^{-\beta S_{0}\left(\mathbf{R}_{u}, \mathbf{V}^{\prime}\right)}= & e^{\beta \mathbf{Q}_{*} \cdot \mathbf{V}^{\prime}} \frac{1}{Z_{v}} \int d \mathbf{R}_{v} e^{-\beta\left[U_{0}(\mathbf{R})+\mathbf{Q}_{*} \cdot \mathbf{V}(\mathbf{R})\right]} \\
& \times \delta\left(\mathbf{V}^{\prime}-\mathbf{V}(\mathbf{R})\right)=e^{\beta \mathbf{Q}_{*} \cdot \mathbf{V}^{\prime}} e^{-\beta S_{*}\left(\mathbf{R}_{u}, \mathbf{V}^{\prime}\right)},
\end{aligned}
$$

where we have attached arbitrary reference charges $\mathbf{Q}_{*}$ $=\mathbf{Q}_{*}\left(\mathbf{R}_{u}\right)$ to the solute sites, and also defined a charged (or shifted) self-energy of the solvent $S_{*}\left(\mathbf{R}_{u}, \mathbf{V}^{\prime}\right)$ by the second equality. Combining the above expression with $E\left(\mathbf{R}_{u}, \mathbf{V}^{\prime}\right)$ gives

$$
F\left(\mathbf{R}_{u}, \mathbf{V}^{\prime}\right)=E\left(\mathbf{R}_{u}, \mathbf{V}^{\prime}\right)-\mathbf{Q}_{*} \cdot \mathbf{V}^{\prime}+S_{*}\left(\mathbf{R}_{u}, \mathbf{V}^{\prime}\right),
$$

where a certain amount of solute-solvent electrostatic coupling is represented by the shifted self-energy. Following the previous section, one may invoke a Gaussian fluctuation model for $S_{*}\left(\mathbf{R}_{u}, \mathbf{V}^{\prime}\right)$ to have

$$
\begin{aligned}
S_{*}\left(\mathbf{R}_{u}, \mathbf{V}^{\prime}\right) \simeq & \frac{1}{2 \beta}\left(\mathbf{V}^{\prime}-\langle\mathbf{V}\rangle_{*}\right) \cdot \mathbf{C}_{*}^{-1} \cdot\left(\mathbf{V}^{\prime}-\langle\mathbf{V}\rangle_{*}\right)+\Delta \mu_{*} \\
& +\delta F\left(\mathbf{C}_{*}\right),
\end{aligned}
$$

where

$$
\begin{aligned}
& \langle\mathbf{V}\rangle_{*}=\langle\mathbf{V}(\mathbf{R})\rangle_{*}, \\
& \mathbf{C}_{*}=\left\langle\left[\mathbf{V}(\mathbf{R})-\langle\mathbf{V}\rangle_{*}\right]\left[\mathbf{V}(\mathbf{R})-\langle\mathbf{V}\rangle_{*}\right]^{T}\right\rangle_{*},
\end{aligned}
$$

with the average $\langle\cdots\rangle_{*}$ defined by

$$
\langle\cdots\rangle_{*}=\frac{\int d \mathbf{R}_{v} e^{-\beta\left[U_{0}(\mathbf{R})+\mathbf{Q}_{*} \cdot \mathbf{V}(\mathbf{R})\right]}(\cdots)}{\int d \mathbf{R}_{v} e^{-\beta\left[U_{0}(\mathbf{R})+\mathbf{Q}_{*} \cdot \mathbf{V}(\mathbf{R})\right]}},
$$

and $\Delta \mu_{*}$ in Eq. (A13) is the solvation free energy of the charged solute defined by

$$
e^{-\beta \Delta \mu_{*}}=\frac{1}{Z_{v}} \int d \mathbf{R}_{v} e^{-\beta\left[U_{0}(\mathbf{R})+\mathbf{Q}_{*} \cdot \mathbf{V}(\mathbf{R})\right]} .
$$

Now that $S_{*}\left(\mathbf{R}_{u}, \mathbf{V}^{\prime}\right)$ is approximated quadratically as in Eq. (A13), it is appealing to expand $E\left(\mathbf{R}_{u}, \mathbf{V}^{\prime}\right)$ in Eq. (A12) also up to second order in $\mathbf{V}^{\prime}$ so that one obtains an overall quadratic expansion of $F\left(\mathbf{R}_{u}, \mathbf{V}^{\prime}\right)$ with respect to $\mathbf{V}^{\prime}$. To do so, let us assume that the reference charge $\mathbf{Q}_{*}$ is derived from a reference external potential $\mathbf{V}_{*}$ such that $\mathbf{Q}_{*}$ $\equiv \mathbf{Q}\left[\Psi\left(\mathbf{V}_{*}\right)\right]$; i.e., the independent variables are now $\mathbf{V}_{*}$ rather than $\mathbf{Q}_{*}$. Expanding $E\left(\mathbf{R}_{u}, \mathbf{V}^{\prime}\right)$ with respect to $\mathbf{V}^{\prime}$ about $\mathbf{V}_{*}$ gives $^{33,53}$

$$
\begin{aligned}
E\left(\mathbf{R}_{u}, \mathbf{V}^{\prime}\right) \simeq & E\left(\mathbf{R}_{u}, \mathbf{V}_{*}\right)+\mathbf{Q}_{*} \cdot\left(\mathbf{V}^{\prime}-\mathbf{V}_{*}\right) \\
& +\frac{1}{2}\left(\mathbf{V}^{\prime}-\mathbf{V}_{*}\right) \cdot \mathbf{K}\left(\mathbf{V}_{*}\right) \cdot\left(\mathbf{V}^{\prime}-\mathbf{V}_{*}\right),
\end{aligned}
$$

where we have used the relation

$$
\frac{\partial E\left(\mathbf{R}_{u}, \mathbf{V}^{\prime}\right)}{\partial \mathbf{V}^{\prime}}=\mathbf{Q}\left[\Psi\left(\mathbf{V}^{\prime}\right)\right]
$$

which is obtained by applying the Hellmann-Feynman theorem to Eq. (6), and

$$
\mathbf{K}\left(\mathbf{V}^{\prime}\right) \equiv \frac{\partial^{2} E\left(\mathbf{R}_{u}, \mathbf{V}^{\prime}\right)}{\partial \mathbf{V}^{\prime} \partial \mathbf{V}^{\prime}}=\frac{\partial \mathbf{Q}\left[\Psi\left(\mathbf{V}^{\prime}\right)\right]}{\partial \mathbf{V}^{\prime}},
$$

which is a type of polarizability matrix called the charge response kernel (CRK). ${ }^{31-33}$ Inserting the quadratic expansions in Eqs. (A13) and (A18) and into Eq. (A12) and rearranging terms, we have

$$
\begin{aligned}
F\left(\mathbf{R}_{u}, \mathbf{V}^{\prime}\right) \simeq & \left\langle\Psi\left(\mathbf{V}_{*}\right)\left|\hat{H}_{0}\right| \Psi\left(\mathbf{V}_{*}\right)\right\rangle+\Delta \mu_{*}+\delta F\left(\mathbf{C}_{*}\right) \\
& +\frac{1}{2}\left(\mathbf{V}^{\prime}-\mathbf{V}_{*}\right) \cdot \mathbf{K}\left(\mathbf{V}_{*}\right) \cdot\left(\mathbf{V}^{\prime}-\mathbf{V}_{*}\right) \\
& +\frac{1}{2 \beta}\left(\mathbf{V}^{\prime}-\langle\mathbf{V}\rangle_{*}\right) \cdot \mathbf{C}_{*}^{-1} \cdot\left(\mathbf{V}^{\prime}-\langle\mathbf{V}\rangle_{*}\right) .
\end{aligned}
$$

Although $F\left(\mathbf{R}_{u}, \mathbf{V}^{\prime}\right)$ has been expanded quadratically in $\mathbf{V}^{\prime}$, the stationary point of this function is neither $\mathbf{V}_{*}$ nor $\langle\mathbf{V}\rangle_{*}$ because $\mathbf{V}_{*}$ (i.e., the input parameter) is not guaranteed to be identical to $\langle\mathbf{V}\rangle_{*}$ (the response field). This in turn suggests that one may choose $\mathbf{V}_{*}$ so that

$$
\mathbf{V}_{*}=\langle\mathbf{V}\rangle_{*},
$$

is satisfied, which results in the so-called self-consistent reaction field. We will denote such a reaction field as $\mathbf{V}_{\text {eq }}\left(\mathbf{R}_{u}\right)$, since it gives the equilibrium point of $F\left(\mathbf{R}_{u}, \mathbf{V}^{\prime}\right)$ at a given $\mathbf{R}_{u}$. Now letting $\mathbf{V}_{*}=\mathbf{V}_{\text {eq }}\left(\mathbf{R}_{u}\right)$, one obtains the following second-order expansion of $F\left(\mathbf{R}_{u}, \mathbf{V}^{\prime}\right)$ :

$$
\begin{aligned}
F\left(\mathbf{R}_{u}, \mathbf{V}^{\prime}\right)= & \widetilde{F}_{\mathrm{eq}}\left(\mathbf{R}_{u}\right)+\frac{1}{2}\left(\mathbf{V}^{\prime}-\mathbf{V}_{\mathrm{eq}}\right) \\
& \cdot\left(\mathbf{K}_{\mathrm{eq}}+\frac{1}{\beta} \mathbf{C}_{\mathrm{eq}}^{-1}\right) \cdot\left(\mathbf{V}^{\prime}-\mathbf{V}_{\mathrm{eq}}\right),
\end{aligned}
$$

where $\widetilde{F}_{\text {eq }}\left(\mathbf{R}_{u}\right)$ is defined by

$$
\tilde{F}_{\mathrm{eq}}\left(\mathbf{R}_{u}\right)=\left\langle\Psi_{\mathrm{eq}}\left|\hat{H}_{0}\right| \Psi_{\mathrm{eq}}\right\rangle+\Delta \mu_{\mathrm{eq}}+\delta F\left(\mathbf{C}_{\mathrm{eq}}\right),
$$

while other quantities having the suffix "eq" are those evaluated at $\mathbf{V}_{*}=\mathbf{V}_{\text {eq }}\left[\right.$ e.g., $\left.\Psi_{\text {eq }}=\Psi\left(\mathbf{V}_{\text {eq }}\right)\right]$. We note that $F_{\text {eq }}\left(\mathbf{R}_{u}\right)$ in Eq. (A24) can be written equivalently (within the quadratic approximation) as

$$
e^{-\beta \tilde{F}_{\mathrm{eq}}\left(\mathbf{R}_{u}\right)}=\frac{1}{Z_{v}} \int d \mathbf{R}_{v} e^{-\beta U\left[\mathbf{R} ; \Psi_{\mathrm{eq}}\right]} \delta\left(\mathbf{V}_{\mathrm{eq}}\left(\mathbf{R}_{u}\right)-\mathbf{V}(\mathbf{R})\right),
$$

where the solvent distribution is constrained with the condition $\mathbf{V}(\mathbf{R})=\mathbf{V}_{\text {eq }}\left(\mathbf{R}_{u}\right)$, while the solute wave function is fixed at $\Psi_{\text {eq }}=\Psi\left(\mathbf{V}_{\text {eq }}\left(\mathbf{R}_{u}\right)\right)$. One can also define an alternative form of equilibrium free energy, 


$$
e^{-\beta F_{\mathrm{eq}}\left(\mathbf{R}_{u}\right)}=\frac{1}{Z_{v}} \int d \mathbf{R}_{v} e^{-\beta U\left[\mathbf{R} ; \Psi_{\mathrm{eq}}\right]},
$$

where the constraint on $\mathbf{V}(\mathbf{R})$ has been eliminated from Eq. (A25). Within the quadratic approximation, the two definitions are related simply by

$$
\widetilde{F}_{\text {eq }}\left(\mathbf{R}_{u}\right)=F_{\text {eq }}\left(\mathbf{R}_{u}\right)+\delta F\left(\mathbf{C}_{\text {eq }}\right) .
$$

Thus, it is a matter of convenience which of Eqs. (A25) and (A26) to use in practice. Furthermore, it is probably a good approximation to regard $\delta F\left(\mathbf{C}_{\text {eq }}\right)$ as constant since it originates from the pre-exponential factor of a Gaussian distribution function and is likely to be a more slowly varying function of $\mathbf{R}_{u}$ than $F_{\text {eq }}\left(\mathbf{R}_{u}\right)$ or $\widetilde{F}_{\text {eq }}\left(\mathbf{R}_{u}\right)$.

It is now interesting to see how the solvation free energy defined in the $\mathrm{QM} / \mathrm{MM}$ sense,

$$
\begin{aligned}
e^{-\beta F_{\mathrm{QM} / \mathrm{MM}}\left(\mathbf{R}_{u}\right)} & \equiv \frac{1}{Z_{v}} \int d \mathbf{R}_{v} e^{-\beta U[\mathbf{R} ; \Psi(\mathbf{V}(\mathbf{R}))]} \\
& =\int d \mathbf{V}^{\prime} e^{-\beta F\left(\mathbf{R}_{u}, \mathbf{V}^{\prime}\right)},
\end{aligned}
$$

relates to $F_{\text {eq }}\left(\mathbf{R}_{u}\right)$ in Eq. (A26), where the second equality follows from Eq. (10). Note that in $F_{\mathrm{QM} / \mathrm{MM}}\left(\mathbf{R}_{u}\right)$ the solute wave function is allowed to fluctuate according to the instantaneous value of the external potential, while in $F_{\text {eq }}\left(\mathbf{R}_{u}\right)$ the wave function is fixed at $\Psi_{\text {eq }}$. The effect of the electronic distortion of $\Psi(\mathbf{V}(\mathbf{R}))$ from $\Psi_{\text {eq }}$ can be quantified by inserting the second-order expansion in Eq. (A23) into the above equation and integrating over $\mathbf{V}^{\prime}$ to give

$$
F_{\mathrm{QM} / \mathrm{MM}}\left(\mathbf{R}_{u}\right)=F_{\mathrm{eq}}\left(\mathbf{R}_{u}\right)-\beta^{-1} \ln \left[\frac{\operatorname{det} \mathbf{C}_{\mathrm{eq}}^{-1}}{\operatorname{det}\left(\mathbf{C}_{\mathrm{eq}}^{-1}+\beta \mathbf{K}_{\mathrm{eq}}\right)}\right]^{1 / 2} .
$$

Setting $\mathbf{K}_{\mathrm{eq}}=0$ (i.e., neglecting the electronic distortion from $\Psi_{\text {eq }}$ or assuming that the eigenvalues of $\beta \mathbf{K}_{\mathrm{eq}}$ are much smaller than those of $\mathbf{C}_{\mathrm{eq}}^{-1}$ ) results in a simple relation $F_{\mathrm{QM} / \mathrm{MM}}\left(\mathbf{R}_{u}\right)=F_{\mathrm{eq}}\left(\mathbf{R}_{u}\right)$, as expected from the definitions in Eqs. (A26) and (A28).

\section{APPENDIX B: INTERPOLATION OF EXTERNAL POTENTIAL}

While the probe-point approximation is quite simple, its accuracy may not be sufficient when the proton is delocalized, e.g., over a double-well potential. An idea for improving the accuracy is to calculate the external potential acting on the proton via interpolation of the external field acting on nearby solute atoms. In the simplest case one may perform a linear interpolation as

$$
V\left(\mathbf{r}_{p} ; \mathbf{R}_{v}\right) \simeq \frac{R\left(\mathrm{HO}^{\prime}\right) V(\mathrm{O})+R(\mathrm{OH}) V\left(\mathrm{O}^{\prime}\right)}{R(\mathrm{OH})+R\left(\mathrm{HO}^{\prime}\right)},
$$

where $\mathrm{O}, \mathrm{H}$, and $\mathrm{O}^{\prime}$ are atoms that constitute a hydrogenbond interface, while $V(\mathrm{O})$ and $V\left(\mathrm{O}^{\prime}\right)$ are external potentials acting on $\mathrm{O}$ and $\mathrm{O}^{\prime}$, respectively. Note that $V(\mathrm{H})$ is not used explicitly but the proton coordinate $\mathbf{r}_{p}$ does appear in Eq. (B1), so the nature of the approximation is somewhat differ- ent from the probe-point approximation. With any interpolation schemes like Eq. (B1), the external-potential vector $\mathbf{V}(\mathbf{R})$ becomes a function only of the solute coordinates $\mathbf{R}_{u}$ and the external potential acting on the solute sites except for the proton, $\tilde{\mathbf{V}}(\tilde{\mathbf{R}})$, which is denoted here as

$$
\mathbf{V}(\mathbf{R}) \simeq \mathbf{V}_{\mathrm{IP}}\left(\mathbf{R}_{u}, \tilde{\mathbf{V}}(\tilde{\mathbf{R}})\right) .
$$

Now, making the above approximation to $\mathbf{V}(\mathbf{R})$ and inserting the resolution of unity $1=\int d \widetilde{\mathbf{V}}^{\prime} \delta\left(\tilde{\mathbf{V}}^{\prime}-\tilde{\mathbf{V}}(\tilde{\mathbf{R}})\right)$ into Eq. (28), one obtains the following rate expression:

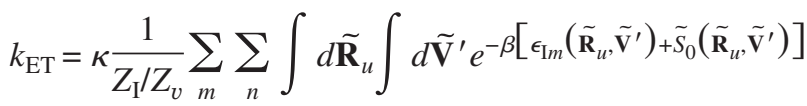

$$
\begin{aligned}
& \times\left|T_{m n}\left(\tilde{\mathbf{R}}_{u}, \tilde{\mathbf{V}}^{\prime}\right)\right|^{2} \delta\left[\epsilon_{\mathrm{Im}}\left(\tilde{\mathbf{R}}_{u}, \tilde{\mathbf{V}}^{\prime}\right)-\epsilon_{\mathrm{IIn}}\left(\tilde{\mathbf{R}}_{u}, \tilde{\mathbf{V}}^{\prime}\right)\right],
\end{aligned}
$$

where $\epsilon_{K l}$ and $\varphi_{K l}$ are defined via

$$
\begin{aligned}
& \hat{h}_{K}\left(\mathbf{V}_{\mathrm{IP}}\left(\mathbf{R}_{u}, \widetilde{\mathbf{V}}^{\prime}\right)\right)_{\varphi_{K l}}\left(\mathbf{r}_{p} ; \widetilde{\mathbf{R}}_{u}, \tilde{\mathbf{V}}^{\prime}\right) \\
& =\epsilon_{K l}\left(\tilde{\mathbf{R}}_{u}, \tilde{\mathbf{V}}^{\prime}\right)_{\varphi_{K l}}\left(\mathbf{r}_{p} ; \tilde{\mathbf{R}}_{u}, \tilde{\mathbf{V}}^{\prime}\right),
\end{aligned}
$$

while $\widetilde{S}_{0}$ is given by

$$
e^{-\beta \tilde{S}_{0}\left(\tilde{\mathbf{R}}_{u}, \tilde{\mathbf{V}}^{\prime}\right)}=\frac{1}{Z_{v}} \int d \mathbf{R}_{v} e^{-\beta U_{0}(\tilde{\mathbf{R}})} \delta\left(\tilde{\mathbf{V}}^{\prime}-\tilde{\mathbf{V}}(\tilde{\mathbf{R}})\right) .
$$

The above interpolation scheme is as simple as the probepoint approximation but can be more accurate by including the spatial variation in the electrostatic potential around the transferring proton.

${ }^{1}$ R. M. Levy, M. Belhadj, and D. B. Kitchen, J. Chem. Phys. 95, 3627 (1991).

${ }^{2}$ R. M. Levy and E. Gallicchio, Annu. Rev. Phys. Chem. 49, 531 (1998).

${ }^{3}$ M. V. Basilevsky and G. E. Chudinov, Chem. Phys. 157, 327 (1991).

${ }^{4}$ M. V. Basilevsky, G. E. Chudinov, and M. D. Newton, Chem. Phys. 179, 263 (1994).

${ }^{5}$ M. V. Vener, I. V. Leontyev, Y. A. Dyakov, M. V. Basilevsky, and M. D. Newton, J. Phys. Chem. B 106, 13078 (2002).

${ }^{6}$ Z. Lu and W. Yang, J. Chem. Phys. 121, 89 (2004).

${ }^{7}$ See also M. Wang, Z. Lu, and W. Yang, J. Chem. Phys. 121, 101 (2004); 124, 124516 (2006).

${ }^{8}$ S. Yamazaki and S. Kato, J. Chem. Phys. 123, 114510 (2005).

${ }^{9}$ B. U. Felderhof, J. Chem. Phys. 67, 493 (1977).

${ }^{10}$ R. A. Marcus, J. Phys. Chem. 98, 7170 (1994).

${ }^{11}$ D. F. Calef and P. G. Wolynes, J. Phys. Chem. 87, 3387 (1983); J. Chem. Phys. 78, 470 (1983).

${ }^{12}$ S. Lee and J. T. Hynes, J. Chem. Phys. 88, 6853 (1988).

${ }^{13}$ H. J. Kim and J. T. Hynes, J. Chem. Phys. 93, 5194 (1990).

${ }^{14}$ J. M. Mayer, Annu. Rev. Phys. Chem. 55, 363 (2004).

${ }^{15}$ S. Y. Reece, J. M. Hodgkiss, J. Stubbe, and D. G. Nocera, Philos. Trans. R. Soc. London, Ser. B 361, 1351 (2006).

${ }^{16}$ J. M. Mayer, D. A. Hrovat, J. L. Thomas, and W. T. Borden, J. Am. Chem. Soc. 124, 11142 (2002).

${ }^{17}$ J. H. Skone, A. V. Soudackov, and S. Hammes-Schiffer, J. Am. Chem. Soc. 128, 16655 (2006).

${ }^{18}$ R. I. Cukier, J. Phys. Chem. 100, 15428 (1996).

${ }^{19}$ R. I. Cukier and D. G. Nocera, Annu. Rev. Phys. Chem. 49, 337 (1998).

${ }^{20}$ R. I. Cukier, Biochim. Biophys. Acta 1655, 37 (2004).

${ }^{21}$ A. V. Soudackov and S. Hammes-Schiffer, J. Chem. Phys. 113, 2385 (2000)

${ }^{22}$ A. V. Soudackov and S. Hammes-Schiffer, J. Am. Chem. Soc. 121, 10598 (1999).

${ }^{23}$ E. Hatcher, A. V. Soudackov, and S. Hammes-Schiffer, J. Am. Chem. Soc. 126, 5763 (2004).

${ }^{24}$ E. Hatcher, A. V. Soudackov, and S. Hammes-Schiffer, J. Am. Chem. Soc. 129, 187 (2007) 
${ }^{25}$ J. L. Cape, M. K. Bowman, and D. M. Kramer, J. Am. Chem. Soc. 127, 4208 (2005).

${ }^{26}$ S. Nagaoka, Y. Nishioku, and K. Mukai, Chem. Phys. Lett. 287, 70 (1998); S. Nagaoka et al., J. Phys. Chem. B 104, 856 (2000).

${ }^{27}$ S. Pressé and R. Silbey, J. Chem. Phys. 124, 164504 (2006).

${ }^{28}$ S. Ten-no, F. Hirata, and S. Kato, J. Chem. Phys. 100, 7443 (1994).

${ }^{29}$ Molecular Theory of Solvation, edited by F. Hirata (Kluwer, Netherlands, 2003).

${ }^{30}$ C. I. Bayly, P. Cieplak, W. D. Cornell, and P. A. Kollman, J. Phys. Chem. 97, 10269 (1993).

${ }^{31}$ A. Morita and S. Kato, J. Am. Chem. Soc. 119, 4021 (1997).

${ }^{32}$ A. Morita and S. Kato, J. Chem. Phys. 108, 6809 (1998).

${ }^{33}$ T. Ishida and A. Morita, J. Chem. Phys. 125, 074112 (2006).

${ }^{34}$ In practice, we make the argument of the delta function in Eq. (10) dimensionless as $\delta\left[\left(\mathbf{V}^{\prime}-\mathbf{V}(\mathbf{R})\right) / \phi_{0}\right]$, where $\phi_{0}$ is an arbitrary unit of electrostatic potential, e.g., $\phi_{0}=1 \mathrm{~V}$. However, we omit this factor throughout for notational simplicity (see also Ref. 44).

${ }^{35} Z_{v}$ is included so that $F\left(\mathbf{R}_{u}, \mathbf{V}^{\prime}\right)$ has a well-defined thermodynamic limit.

${ }^{36}$ V. May and O. Kuhn, Charge and Energy Transfer Dynamics in Molecular Systems (Wiley, Berlin, 2000).

${ }^{37}$ J.-K. Hwang and A. Warshel, J. Am. Chem. Soc. 109, 715 (1987).

${ }^{38}$ G. King and A. Warshel, J. Chem. Phys. 93, 8682 (1990).

${ }^{39}$ E. A. Carter and J. T. Hynes, J. Phys. Chem. 93, 2184 (1989).

${ }^{40}$ R. A. Kuharski, J. S. Bader, D. Chandler, M. Sprik, M. L. Klein, and R. W. Impey, J. Chem. Phys. 89, 3248 (1988).
${ }^{41}$ A. Yoshimori, T. Kakitani, Y. Enomoto, and N. Mataga, J. Phys. Chem. 93, 8316 (1989).

${ }^{42}$ M. Tachiya, J. Phys. Chem. 93, 7050 (1989); 97, 5911 (1993).

${ }^{43}$ H.-X. Zhou and A. Szabo, J. Chem. Phys. 103, 3481 (1995).

${ }^{44}$ J. Blumberger and M. Sprik, J. Phys. Chem. B 109, 6793 (2005)

${ }^{45}$ P. P. Schmidt, J. Chem. Phys. 58, 4384 (1973).

${ }^{46}$ R. A. Marcus, J. Chem. Phys. 81, 4494 (1984).

${ }^{47}$ J. Espinosa-Garcia and C. Gutierrez-Merino, J. Phys. Chem. A 107, 9712 (2003).

${ }^{48}$ T. H. Dunning, Jr. and P. J. Hay, in Methods of Electronic Structure Theory, edited by H. F. Schaefer III (Plenum, New York, 1977).

${ }^{49}$ M. W. Schmidt et al., J. Comput. Chem. 14, 1347 (1993).

${ }^{50}$ W. L. Jorgensen and J. M. Briggs, Mol. Phys. 63, 547 (1988).

${ }^{51}$ W. D. Cornell et al., J. Am. Chem. Soc. 117, 5179 (1995).

${ }^{52}$ See M. Higashi and S. Kato, J. Phys. Chem. A 109, 9867 (2005) for the MP2 correction of the equilibrium free energy.

${ }^{53}$ K. Naka, A. Morita, and S. Kato, J. Chem. Phys. 110, 3484 (1999).

${ }^{54}$ More precisely, Fig. 8 was obtained by calculating $E_{K}\left(\mathbf{r}_{p}, \mathbf{X}^{\ddagger}\right)$ rather than $F_{K}\left(\mathbf{r}_{p}, \mathbf{X}^{\ddagger}\right)$ as a function of $x_{p}$ by assuming the probe-point approximation (i.e., solvent self-energy is constant as a function of $\mathbf{r}_{p}$ ).

${ }^{55}$ R. P. Bell, The Tunnel Effect in Chemistry (Chapman and Hall, New York, 1980).

${ }^{56} \Delta \mu_{0}$ can be calculated in various approximate ways, e.g., by using a linear-response approximation (Ref. 8).

${ }^{57}$ J. Aqvist and T. Hansson, J. Phys. Chem. 100, 9512 (1996). 